Article

\title{
Manganiakasakaite-(La) and Ferriakasakaite-(Ce), Two New Epidote Supergroup Minerals from Piedmont, Italy
}

\author{
Cristian Biagioni ${ }^{1, *}$, Paola Bonazzi ${ }^{2}$, Marco Pasero ${ }^{1}$, Federica Zaccarini ${ }^{3}$, Corrado Balestra ${ }^{4}$, \\ Roberto Bracco ${ }^{5}$ and Marco E. Ciriotti ${ }^{6}$ \\ 1 Dipartimento di Scienze della Terra, Università di Pisa, Via Santa Maria 53, I-56126 Pisa, Italy; \\ marco.pasero@unipi.it \\ 2 Dipartimento di Scienze della Terra, Università di Firenze, Via G. La Pira 4, I-50132 Firenze, Italy; \\ paola.bonazzi@unifi.it \\ 3 Department of Applied Geological Sciences and Geophysics, University of Leoben, Peter Tunner Str. 5, \\ A-8700 Leoben, Austria; Federica.Zaccarini@unileoben.ac.at \\ 4 Associazione Micromineralogica Italiana, Via Luigi Delfino 74, I-17017 Millesimo (SV), Italy; \\ c.balestra@filmferrania.it \\ 5 Associazione Micromineralogica Italiana, Via Montenotte 18/6, I-17100 Savona, Italy; woof_59@yahoo.it \\ 6 Associazione Micromineralogica Italiana, Via San Pietro 55, I-10073 Devesi-Ciriè (TO), Italy; m.ciriotti@tin.it \\ * Correspondence: cristian.biagioni@unipi.it; Tel.: +39-050-221-5789
}

Received: 20 May 2019; Accepted: 7 June 2019; Published: 9 June 2019

Abstract: Two new monoclinic $\left(P 2_{1} / m\right)$ epidote supergroup minerals manganiakasakaite-(La) and ferriakasakaite-(Ce) were found in the small Mn ore deposit of Monte Maniglia, Bellino, Varaita Valley, Cuneo Province, Piedmont, Italy. Manganiakasakaite-(La) occurs as subhedral grains embedded in pyroxmangite. Its empirical formula is ${ }^{A(1)}\left(\mathrm{Ca}_{0.62} \mathrm{Mn}^{2+}{ }_{0.38}\right){ }^{A(2)}\left(\mathrm{La}_{0.52} \mathrm{Nd}_{0.08} \operatorname{Pr}_{0.07} \mathrm{Ce}_{0.07} \mathrm{Y}_{0.01} \mathrm{Ca}_{0.25}\right)$ ${ }^{M(1)}\left(\mathrm{Mn}^{3+}{ }_{0.52} \mathrm{Fe}^{3+}{ }_{0.28} \mathrm{Al}_{0.18} \mathrm{~V}^{3+}{ }_{0.01}\right)^{M(2)} \mathrm{Al}_{1.00}{ }^{M(3)}\left(\mathrm{Mn}^{2+}{ }_{0.60} \mathrm{Mn}^{3+}{ }_{0.27} \mathrm{Mg}_{0.13}\right)^{T(1-3)}\left(\mathrm{Si}_{2.99} \mathrm{Al}_{0.01}\right) \mathrm{O}_{12}$ $(\mathrm{OH})$, corresponding to the end-member formula CaLaMn ${ }^{3+} \mathrm{AlMn}^{2+}\left(\mathrm{Si}_{2} \mathrm{O}_{7}\right)\left(\mathrm{SiO}_{4}\right) \mathrm{O}(\mathrm{OH})$. Unit-cell parameters are $a=8.9057(10), b=5.7294(6), c=10.1134(11) \AA, \beta=113.713(5)^{\circ}, V=472.46(9) \AA^{3}, Z=2$. The crystal structure of manganiakasakaite-(La) was refined to a final $R_{1}=0.0262$ for 2119 reflections with $F_{\mathrm{o}}>4 \sigma\left(F_{\mathrm{o}}\right)$ and 125 refined parameters. Ferriakasakaite-(Ce) occurs as small homogeneous domains within strongly inhomogeneous prismatic crystals, where other epidote supergroup minerals coexist [manganiandrosite-(Ce), "androsite-(Ce)", and epidote]. Associated minerals are calcite and hematite. Its empirical formula is ${ }^{A(1)}\left(\mathrm{Ca}_{0.64} \mathrm{Mn}^{2+}{ }_{0.36}\right){ }^{A(2)}\left(\mathrm{Ce}_{0.37} \mathrm{La}_{0.17} \mathrm{Nd}_{0.06} \operatorname{Pr}_{0.03} \mathrm{Ca}_{0.35} \square_{0.02}\right)$ ${ }^{M(1)}\left(\mathrm{Fe}^{3+}{ }_{0.61} \mathrm{Al}_{0.39}\right){ }^{M(2)} \mathrm{Al}_{1.00}{ }^{M(3)}\left(\mathrm{Mn}^{2+}{ }_{0.64} \mathrm{Mn}^{3+}{ }_{0.33} \mathrm{Fe}^{3+}{ }_{0.02} \mathrm{Mg}_{0.01}\right){ }^{T(1-3)} \mathrm{Si}_{3.01} \mathrm{O}_{12} \quad(\mathrm{OH})$, the end-member formula being $\mathrm{CaCeFe}^{3+} \mathrm{AlMn}^{2+}\left(\mathrm{Si}_{2} \mathrm{O}_{7}\right)\left(\mathrm{SiO}_{4}\right) \mathrm{O}(\mathrm{OH})$. Unit-cell parameters are $a=$ 8.9033(3), $b=5.7066(2), c=10.1363(3) \AA, \beta=114.222(2)^{\circ}, V=469.66(3) \AA^{3}, Z=2$. The crystal structure of ferriakasakaite-(Ce) was refined to a final $R_{1}=0.0196$ for 1960 unique reflections with $F_{\mathrm{o}}>4 \sigma\left(F_{\mathrm{o}}\right)$ and 124 refined parameters.

Keywords: manganiakasakaite-(La); ferriakasakaite-(Ce); epidote supergroup; allanite group; new mineral; Monte Maniglia; Piedmont; Italy

\section{Introduction}

Epidotes are mixed-anion silicates with general formula $A_{2} M_{3}\left[T_{2} \mathrm{O}_{7}\right]\left[T \mathrm{O}_{4}\right](\mathrm{O}, \mathrm{F})(\mathrm{OH}, \mathrm{O})[1]$ which typically occur as rock-forming minerals in low- to medium-grade metamorphic rocks, in hydrothermal settings, or as accessory phases in magmatic rocks. As of today, the epidote supergroup includes 30 monoclinic mineral species; of them, 17 belong to the allanite group, 10 to the clinozoisite group, 
and 2 to the dollaseite group. Two additional minerals stand alone: zoisite, the unique example of epidote with orthorhombic symmetry, and åskagenite-(Nd), the unique example of epidote having a divalent anion dominant at the $\mathrm{O}(10)$ site. We herewith describe manganiakasakaite-(La) and ferriakasakaite-(Ce), two new minerals belonging to the allanite group. In this group, a trivalent cation, typically a $R E E$, is dominant at the $A(2)$ site, and a divalent cation is dominant at the $M(3)$ site. According to the general guidelines for the nomenclature of epidotes [1], each distinct combination of dominant cations at the $A(1)$ and $M(3)$ sites deserves a proper root-name. In particular, the root-name "akasakaite" (after Masahide Akasaka, professor of mineralogy at the Shimane University) corresponds to the pair $A(1)=\mathrm{Ca}$ and $M(3)=\mathrm{Mn}^{2+}$ [2]. Then, an adjectival prefix is appended to the root-name for the dominant cation at the $M(1)$ (other than $\mathrm{Al}$ ), and a Levinson suffix [3] is appended to the root-name for the dominant REE at the $A(2)$ site. The names of the two new minerals are in keeping with the above guidelines, as they represent chemical variants of the mineral ferriakasakaite-(La), which was first described from Japan [2]. Both manganiakasakaite-(La) and ferriakasakaite-(Ce) were found in the Mn ore deposit of Monte Maniglia, Bellino, Varaita Valley, Cuneo Province, Piedmont, Italy. The geographic coordinates of the type locality are $44^{\circ} 33^{\prime} 42.5^{\prime \prime} \mathrm{N}, 6^{\circ} 54^{\prime} 59.2^{\prime \prime} \mathrm{E}$. The new minerals and their names have been approved by the IMA-CNMNC (IMA \#2017-028 and \#2018-087). Type material has been deposited in the mineralogical collections of the Museo di Storia Naturale, Università di Pisa, Via Roma 79, Calci (PI), Italy, under the catalogue numbers 19907 for manganiakasakaite-(La) and 19903 for ferriakasakaite-(Ce).

\section{Occurrence and Physical Properties}

The small Mn ore deposit of the Monte Maniglia is located along a ridge connecting the Bassa di Terra Rossa (2838 $\mathrm{m}$ above sea level, a.s.1.) and the top of the Monte Maniglia (3177 $\mathrm{m}$ a.s.1.). The ore deposit is hosted in a Mesozoic metasedimentary sequence overlying Permian quartzite, within the so-called Maniglia-type stratigraphic series, belonging to the Acceglio Zone [4]. According to Le Guernic [4], the age of the Mn ore deposit could be Cretaceous.

Previous mineralogical studies allowed the identification of the following mineral species: akatoreite, amphibole group minerals, arseniosiderite, axinite-(Mn), bementite, braunite, calcite, clinochlore, hollandite, malachite, muscovite, neotocite, opal, palenzonaite, piemontite, pyroxmangite, quartz, rhodochrosite, rhodonite, spessartine, and tetrahedrite [5-7].

Manganiakasakaite-(La) was identified as dark brown subhedral grains, up to $0.5 \mathrm{~mm}$ in size, embedded in pyroxmangite (Figure 1a), whereas ferriakasakaite-(Ce) forms dark brown prismatic crystals, elongated on [010], up to $1 \mathrm{~mm}$ in size (Figure 1b), associated with calcite and hematite. Due to the close similarities between these two minerals, their distinction based on physical properties is virtually impossible. Moreover, as will be illustrated below, some grains are strongly zoned with different portions of the crystal requiring different mineral names within the epidote supergroup. The new minerals described here exhibit very similar appearance: they are transparent, brittle, with irregular/uneven to conchoidal fracture, brown streak, and vitreous luster. Fluorescence was not observed. In both cases, hardness was not measured, owing to the small size of homogeneous material. However, a Mohs hardness of 51/2-6 could be estimated based on the average values of other allanite group minerals. For the same reason, density was not measured. The calculated density (on the basis of empirical formulae) for manganiakasakaite-(La) and ferriakasakaite-(Ce) is 4.09 and $4.02 \mathrm{~g} / \mathrm{cm}^{3}$, respectively. Optical properties were not measured because the only suitable grains were embedded in epoxy resin to carry out the electron-microprobe analysis. However, as stated by Armbruster et al. [1], optical data are ambiguous in mineral identification when the chemical compositions are too complex. The mean refractive indices, obtained from the Gladstone-Dale relationship [8,9], using the empirical formula and the calculated density, are 1.860 and 1.830 for manganiakasakaite-(La) and ferriakasakaite-(Ce), respectively. 

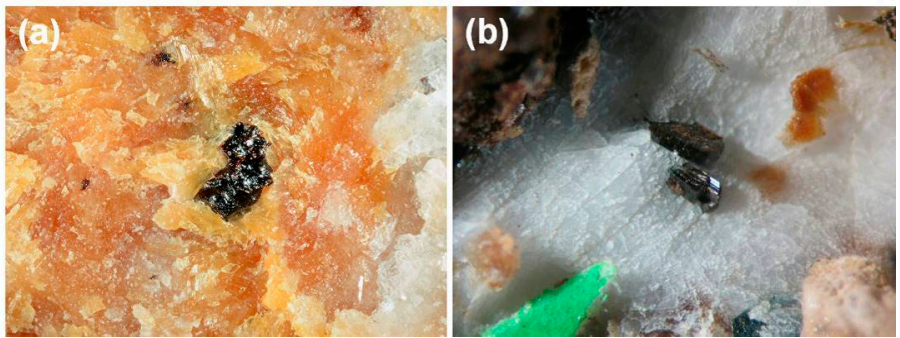

Figure 1. Holotype material of manganiakasakaite-(La) (a) and ferriakasakaite-(Ce). In (a), manganiakasakaite-(La) occurs as a subhedral grain hosted in pyroxmangite. Field of view: $1.5 \times 2 \mathrm{~mm}$. Collection Museo di Storia Naturale, University of Pisa (catalogue number 19907). In (b), ferriakasakaite-(Ce), as prismatic crystals in calcite, up to $1 \mathrm{~mm}$ long. Collection Museo di Storia Naturale, University of Pisa (catalogue number 19903).

\subsection{Chemical Data}

The examined crystals of the epidote supergroup minerals from Monte Maniglia are usually chemically zoned. Whereas the studied grain of manganiakasakaite-(La) was quite homogeneous, the grains sampled from specimen \#19903, where ferriakasakaite-(Ce) was found, are inhomogeneous.

Quantitative chemical analyses for both minerals were carried out using a Superprobe JEOL JXA 8200 electron microprobe (WDS mode, $20 \mathrm{kV}, 10 \mathrm{nA}, 1 \mu \mathrm{m}$ beam diameter) at the Eugen F. Stumpfl laboratory, Leoben University, Austria. Counting times were $20 \mathrm{~s}$ on the peak and $10 \mathrm{~s}$ on the left and right backgrounds. The position of the measurement of backgrounds was carefully selected to avoid overlapping among the analyzed elements. The ZAF correction method was used. Table 1 gives the results of the chemical analyses and the used standards. Fluorine was sought but not detected in manganiakasakaite-(La), whereas $\mathrm{Y}, \mathrm{Ti}, \mathrm{V}$, and $\mathrm{F}$ were sought but not detected in ferriakasakaite-(Ce). No direct $\mathrm{H}_{2} \mathrm{O}$ determination was performed, owing to the scarcity of available material. Similarly, Mössbauer spectra could not be collected due to the limited amount of available samples. Thus, the $\mathrm{Fe}^{2+} / \mathrm{Fe}^{3+}$ and $\mathrm{Mn}^{2+} / \mathrm{Mn}^{3+}$ values were calculated in agreement with the recommendations of Armbruster et al. [1], oxidizing first $\mathrm{Fe}^{2+}$, then $\mathrm{Mn}^{2+}$, in order to account for their different redox potentials. For both manganiakasakaite-(La) and ferriakasakaite-(Ce) all $\mathrm{Fe}$ is in its trivalent state, in keeping with the oxidized nature of the mineral assemblages, characterized by the widespread occurrence of hematite.

Table 1. Chemical composition (in wt \%) of holotype manganiakasakaite-(La) and ferriakasakaite-(Ce).

\begin{tabular}{|c|c|c|c|c|c|c|c|c|}
\hline \multirow[b]{2}{*}{ Constituent } & \multicolumn{4}{|c|}{ Manganiakasakaite-(La) } & \multicolumn{4}{|c|}{ Ferriakasakaite-(Ce) } \\
\hline & $\begin{array}{c}\text { Mean } \\
(n=10)\end{array}$ & Range & e.s.d. ${ }^{1}$ & Standard & $\begin{array}{l}\text { Mean } \\
(n=5)\end{array}$ & Range & e.s.d. ${ }^{1}$ & Standard \\
\hline $\mathrm{SiO}_{2}$ & 30.69 & $29.94-31.79$ & 0.54 & rhodonite & 31.90 & $31.19-32.18$ & 0.41 & bustamite \\
\hline $\mathrm{Al}_{2} \mathrm{O}_{3}$ & 10.39 & $10.16-10.70$ & 0.17 & albite & 12.54 & $12.30-12.93$ & 0.23 & corundum \\
\hline $\mathrm{V}_{2} \mathrm{O}_{3}$ & 0.17 & $0.10-0.29$ & 0.07 & metallic V & - & - & - & - \\
\hline $\mathrm{Fe}_{2} \mathrm{O}_{3}$ & 3.87 & $3.54-4.32$ & 0.26 & olivine & 8.81 & $8.71-8.95$ & 0.09 & chromite \\
\hline $\mathrm{Y}_{2} \mathrm{O}_{3}$ & 0.17 & $0.07-0.28$ & 0.07 & $\mathrm{Y}$-Al garnet & - & - & - & - \\
\hline $\mathrm{La}_{2} \mathrm{O}_{3}$ & 14.61 & $13.16-15.89$ & 0.79 & monazite & 4.89 & $4.65-5.02$ & 0.17 & monazite \\
\hline $\mathrm{Ce}_{2} \mathrm{O}_{3}$ & 1.88 & $1.73-2.09$ & 0.11 & monazite & 10.69 & $10.56-10.82$ & 0.10 & monazite \\
\hline $\mathrm{Pr}_{2} \mathrm{O}_{3}$ & 2.04 & $1.86-2.25$ & 0.12 & synthetic glass & 0.85 & $0.84-0.87$ & 0.02 & synthetic glass \\
\hline $\mathrm{Nd}_{2} \mathrm{O}_{3}$ & 2.21 & $1.76-2.69$ & 0.37 & synthetic glass & 1.82 & $1.72-2.06$ & 0.14 & synthetic glass \\
\hline $\mathrm{Gd}_{2} \mathrm{O}_{3}$ & 0.10 & $0.00-0.24$ & 0.08 & synthetic glass & 0.15 & $0.07-0.21$ & 0.05 & synthetic glass \\
\hline $\mathrm{MgO}$ & 0.88 & $0.77-1.03$ & 0.08 & olivine & 0.09 & $0.07-0.13$ & 0.02 & olivine \\
\hline $\mathrm{CaO}$ & 8.28 & $7.78-8.97$ & 0.38 & wollastonite & 9.82 & $9.67-10.00$ & 0.14 & wollastonite \\
\hline $\mathrm{MnO}_{\text {tot }}$ & 21.58 & $21.30-21.81$ & 0.18 & rhodonite & 16.65 & $16.39-16.81$ & 0.16 & rhodonite \\
\hline $\mathrm{MnO}^{*}$ & 11.98 & - & - & - & 12.56 & - & - & - \\
\hline $\mathrm{Mn}_{2} \mathrm{O}_{3} *$ & 10.69 & - & - & - & 4.56 & - & - & - \\
\hline $\mathrm{H}_{2} \mathrm{O}_{(\text {calc })} * *$ & 1.54 & - & - & - & 1.59 & - & - & - \\
\hline Total & 99.50 & - & - & - & 100.27 & - & - & - \\
\hline
\end{tabular}

Note: ${ }^{1}$ estimated standard deviation; ${ }^{*}$ Calculated in order to yield $8(A+M+T)$ cations and 25 positive charges per formula unit; ${ }^{* *}$ Calculated in order to yield $1 \mathrm{OH}$ group per formula unit. 
The empirical formula of holotype manganiakasakaite-(La) is ${ }^{A(1)}\left(\mathrm{Ca}_{0.62} \mathrm{Mn}^{2+}{ }_{0.38}\right)$ ${ }^{A(2)}\left(\mathrm{La}_{0.52} \mathrm{Nd}_{0.08} \mathrm{Pr}_{0.07} \mathrm{Ce}_{0.07} \mathrm{Y}_{0.01} \mathrm{Ca}_{0.25}\right) \quad M(1)\left(\mathrm{Mn}^{3+}{ }_{0.52} \mathrm{Fe}^{3+}{ }_{0.28} \mathrm{Al}_{0.18} \mathrm{~V}^{3+}{ }_{0.01}\right) \quad{ }^{M(2)} \mathrm{Al}_{1.00}$ $M(3)\left(\mathrm{Mn}^{2+}{ }_{0.60} \mathrm{Mn}^{3+}{ }_{0.27} \mathrm{Mg}_{0.13}\right)^{T(1-3)}\left(\mathrm{Si}_{2.99} \mathrm{Al}_{0.01}\right) \mathrm{O}_{12}(\mathrm{OH})$. The ideal formula of manganiakasakaite-(La) is $\mathrm{CaLaMn}^{3+} \mathrm{AlMn}^{2+}\left(\mathrm{Si}_{2} \mathrm{O}_{7}\right)\left(\mathrm{SiO}_{4}\right) \mathrm{O}(\mathrm{OH})$, which requires (in wt \%): $\mathrm{SiO}_{2} 29.59, \mathrm{Al}_{2} \mathrm{O}_{3} 8.37, \mathrm{Mn}_{2} \mathrm{O}_{3}$ 12.96, $\mathrm{La}_{2} \mathrm{O}_{3}$ 26.75, $\mathrm{CaO} 9.21, \mathrm{MnO} 11.65, \mathrm{H}_{2} \mathrm{O} 1.48$, sum 100.01.

The empirical formula of holotype ferriakasakaite-(Ce) is ${ }^{A(1)}\left(\mathrm{Ca}_{0.64} \mathrm{Mn}^{2+}{ }_{0.36}\right)$ ${ }^{A(2)}\left(\mathrm{Ce}_{0.37} \mathrm{La}_{0.17} \mathrm{Nd}_{0.06} \operatorname{Pr}_{0.03} \mathrm{Ca}_{0.35} \square_{0.02}{ }^{M(1)}\left(\mathrm{Fe}^{3+}{ }_{0.61} \mathrm{Al}_{0.39}\right)^{M(2)} \mathrm{Al}_{1.00}{ }^{M(3)}\left(\mathrm{Mn}^{2+}{ }_{0.64} \mathrm{Mn}^{3+}{ }_{0.33} \mathrm{Fe}^{3+}{ }_{0.02} \mathrm{Mg}_{0.01}\right)\right.$ ${ }^{T(1-3)} \mathrm{Si}_{3.01} \mathrm{O}_{12}(\mathrm{OH})$. The ideal formula of ferriakasakaite-(Ce) is $\mathrm{CaCeFe}^{3+} \mathrm{AlMn}^{2+}\left(\mathrm{Si}_{2} \mathrm{O}_{7}\right)(\mathrm{SiO}) \mathrm{O}(\mathrm{OH})$, which requires (in wt \%) $\mathrm{SiO}_{2} 29.49, \mathrm{Al}_{2} \mathrm{O}_{3}$ 8.34, $\mathrm{Fe}_{2} \mathrm{O}_{3}$ 13.06, $\mathrm{Ce}_{2} \mathrm{O}_{3}$ 26.85, $\mathrm{CaO}$ 9.18, $\mathrm{MnO}$ 11.61, $\mathrm{H}_{2} \mathrm{O}$ 1.49, sum 100.00 .

As reported above, ferriakasakaite-(Ce) occurs in specimen \#19903, whose grains are strongly inhomogeneous. Back-scattered electron (BSE) images and X-ray maps allowed to describe such an inhomogeneity (Figures 2 and 3). Sample \#19903-1 is composed by two grains (Figure 2). The larger one is formed by two domains, whereas the smaller one is homogeneous and was used for the collection of chemical data and single-crystal $X$-ray diffraction study of ferriakasakaite-(Ce). The main chemical inhomogeneity is related to the Ca and La contents; indeed, Ca is depleted and La is enriched in one of the domains occurring in the larger grain and characterized by a light grey color in BSE image.
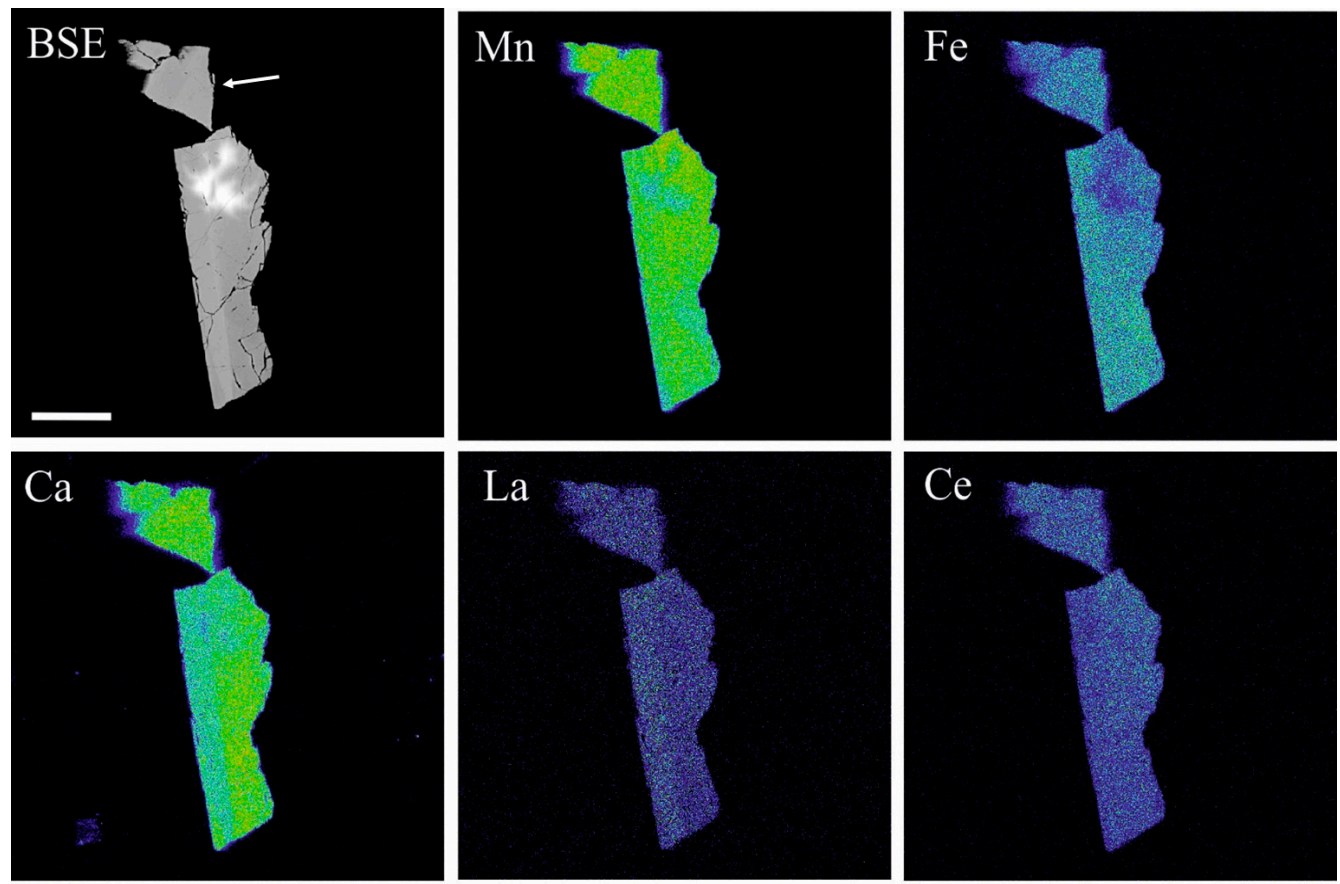

Concentration

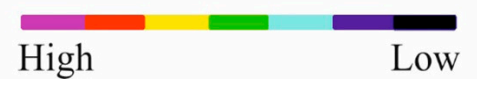

Figure 2. Back-scattered electron (BSE) image and X-ray maps of sample \#19903-1, showing the distribution of $\mathrm{Mn}, \mathrm{Fe}, \mathrm{Ca}, \mathrm{La}$, and $\mathrm{Ce}$. In the BSE image, the bright areas are due to the charging of the surface. The smaller grain used for single-crystal X-ray diffraction is indicated by a white arrow in BSE image. Scale bar $=50 \mu \mathrm{m}$.

Sample \#19903-2 is severely zoned (Figure 3), with three different domains characterized by distinct grey hues. The core is light grey, and it is surrounded by domains having grey (lower left in Figure 3) and light grey (upper right in Figure 3) colors. A dark grey fracture cuts the grain and it is filled by an unidentified Mn silicate (pyroxmangite?). The core is enriched in Mn and REE, whereas the other domains are richer in Fe and Ca. 

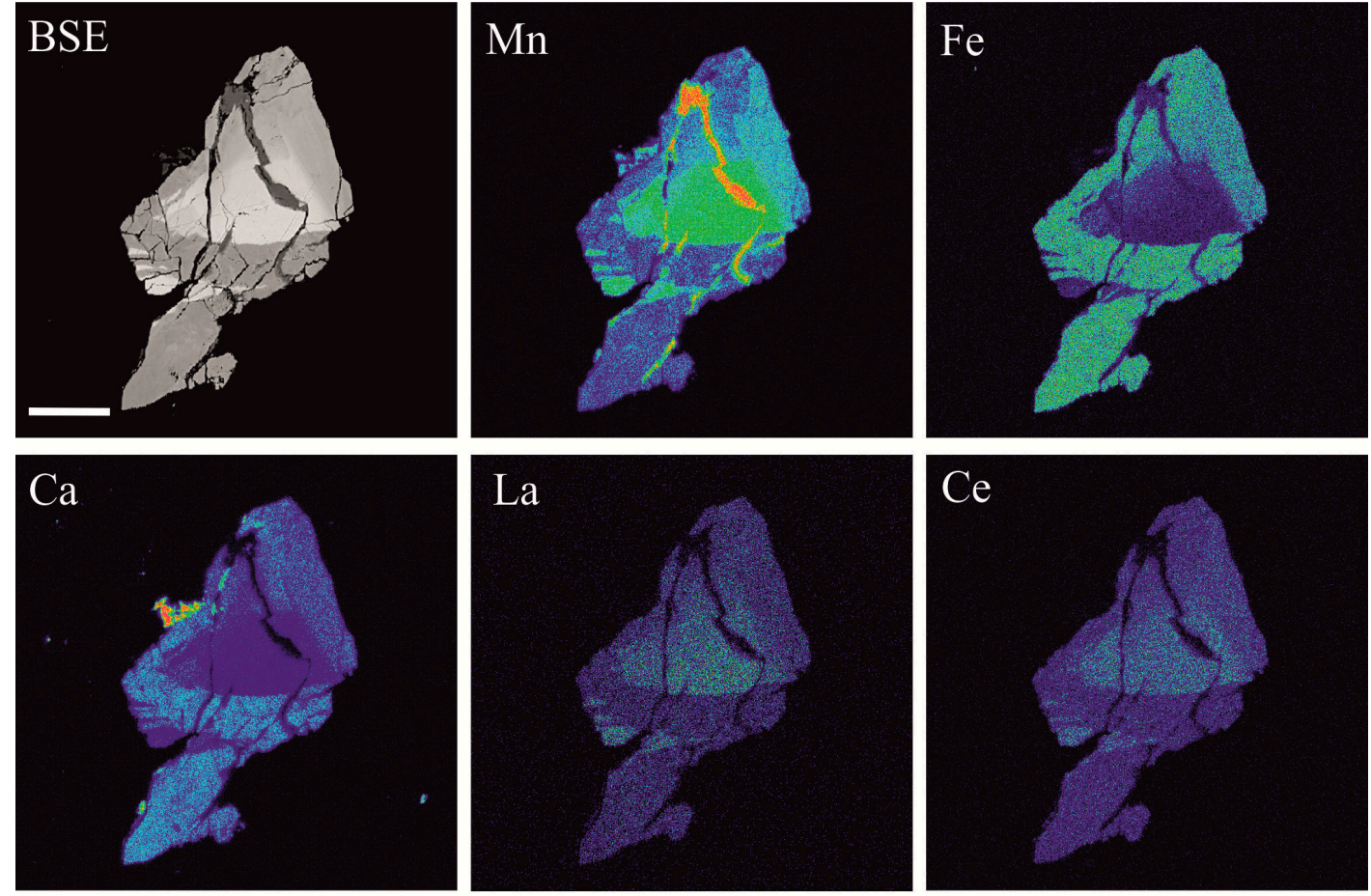

Concentration

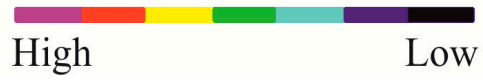

Figure 3. Back-scattered electron (BSE) image and X-ray maps of sample \#19903-2, showing the distribution of $\mathrm{Mn}, \mathrm{Fe}, \mathrm{Ca}, \mathrm{La}$, and Ce. Scale bar $=50 \mu \mathrm{m}$.

Table 2 gives average chemical analyses of the different domains occurring in the two studied samples, with the exception of data of the grain of ferriakasakaite-(Ce) used for single-crystal X-ray diffraction, given in Table 1 .

Table 2. Chemical composition (in wt \%) of ferriakasakaite-(Ce) and coexisting epidote group minerals in samples \#19903-1 and 19903-2.

\begin{tabular}{|c|c|c|c|c|c|}
\hline \multirow[b]{2}{*}{ Constituent } & \multicolumn{2}{|c|}{ Sample \#19903-1 (Large) } & \multicolumn{3}{|c|}{ Sample \#19903-2 } \\
\hline & $\begin{array}{c}\text { Grey } \\
(n=10)\end{array}$ & $\begin{array}{l}\text { Light Grey } \\
\quad(n=3)\end{array}$ & $\begin{array}{l}\text { Light Grey } \\
\quad(n=5)\end{array}$ & $\begin{array}{c}\text { Grey } \\
(n=5)\end{array}$ & $\begin{array}{c}\text { Dark Grey } \\
\quad(n=5)\end{array}$ \\
\hline $\mathrm{SiO}_{2}$ & $32.43(48)$ & $31.86(10)$ & 29.34(29) & $31.40(12)$ & $32.36(48)$ \\
\hline $\mathrm{Al}_{2} \mathrm{O}_{3}$ & $12.85(32)$ & 14.12(88) & $9.88(27)$ & $12.52(62)$ & $14.16(24)$ \\
\hline $\mathrm{Fe}_{2} \mathrm{O}_{3 \text { (tot) }}$ & $9.58(69)$ & $9.26(23)$ & $3.07(68)$ & $9.21(50)$ & $11.88(8)$ \\
\hline $\mathrm{Fe}_{2} \mathrm{O}_{3}$ * & - & 8.70 & - & - & - \\
\hline $\mathrm{FeO}^{*}$ & - & 0.52 & - & - & - \\
\hline $\mathrm{La}_{2} \mathrm{O}_{3}$ & $4.63(23)$ & $4.99(17)$ & $8.79(68)$ & $4.70(24)$ & $3.76(11)$ \\
\hline $\mathrm{Ce}_{2} \mathrm{O}_{3}$ & $10.40(64)$ & $10.74(11)$ & 13.52(42) & $9.86(71)$ & $7.47(27)$ \\
\hline $\mathrm{Pr}_{2} \mathrm{O}_{3}$ & $0.82(4)$ & $0.92(8)$ & $1.49(11)$ & $0.85(2)$ & $0.68(4)$ \\
\hline $\mathrm{Nd}_{2} \mathrm{O}_{3}$ & $1.71(8)$ & $1.33(10)$ & $1.26(10)$ & 2.04(33) & $1.61(9)$ \\
\hline $\mathrm{Gd}_{2} \mathrm{O}_{3}$ & $0.06(3)$ & $0.07(8)$ & $0.05(5)$ & $0.15(7)$ & $0.03(2)$ \\
\hline $\mathrm{MgO}$ & $0.07(2)$ & $0.05(2)$ & $0.12(3)$ & $0.04(2)$ & $0.07(2)$ \\
\hline $\mathrm{CaO}$ & $9.64(42)$ & 7.44(34) & $5.03(25)$ & $8.75(22)$ & $10.41(16)$ \\
\hline $\mathrm{MnO}_{(\mathrm{tot})}$ & 15.82(97) & $15.34(21)$ & $24.86(74)$ & $16.66(119)$ & $12.85(15)$ \\
\hline $\mathrm{MnO}^{*}$ & 13.48 & - & 16.42 & 13.62 & 12.41 \\
\hline $\mathrm{Mn}_{2} \mathrm{O}_{3} *$ & 2.61 & - & 9.39 & 3.38 & 0.48 \\
\hline $\mathrm{H}_{2} \mathrm{O}_{\text {(calc) }} * *$ & 1.60 & 1.56 & 1.49 & 1.55 & 1.60 \\
\hline Total & 99.88 & 97.64 & 99.85 & 98.07 & 96.92 \\
\hline
\end{tabular}

Note: * Calculated in order to yield $8(A+M+T)$ cations and 25 positive charges per formula unit; ${ }^{* *}$ Calculated in order to yield $1 \mathrm{OH}$ group per formula unit. Only average chemical data and estimated standard deviations (within brackets) are given. 
The darker domain in the larger grain of sample \#19903-1 has chemical composition ${ }^{A(1)}\left(\mathrm{Ca}_{0.63} \mathrm{Mn}^{2+}{ }_{0.37}\right)$ ${ }^{A(2)}\left(\mathrm{Ce}_{0.36} \mathrm{La}_{0.16} \mathrm{Nd}_{0.06} \mathrm{Pr}_{0.03} \mathrm{Ca}_{0.34} \square_{0.05}\right)^{M(1)}\left(\mathrm{Fe}^{3+}{ }_{0.58} \mathrm{Al}_{0.42}\right)^{M(2)} \mathrm{Al}_{1.00}{ }^{M(3)}\left(\mathrm{Mn}^{2+}{ }_{0.70} \mathrm{Mn}^{3+}{ }_{0.19} \mathrm{Fe}^{3+}{ }_{0.10} \mathrm{Mg}_{0.01}\right)$ ${ }^{T(1-3)} \mathrm{Si}_{3.05} \mathrm{O}_{12}(\mathrm{OH})$. It matches quite well with the composition of holotype ferriakasakaite-(Ce) (Table 1), with a slightly higher Fe content. On the contrary, the light grey domain, having chemical formula ${ }^{A(1)}\left(\mathrm{Mn}^{2+}{ }_{0.53} \mathrm{Ca}_{0.47}\right)^{A(2)}\left(\mathrm{Ce}_{0.38} \mathrm{La}_{0.18} \mathrm{Nd}_{0.05} \mathrm{Pr}_{0.03} \mathrm{Ca}_{0.30} \square_{0.06}\right)^{M(1)}\left(\mathrm{Al}_{0.60} \mathrm{Fe}^{3+}{ }_{0.40}\right)^{M(2)} \mathrm{Al}_{1.00}$ ${ }^{M(3)}\left(\mathrm{Mn}^{2+}{ }_{0.72} \mathrm{Fe}^{3+}{ }_{0.23} \mathrm{Fe}^{2+}{ }_{0.04} \mathrm{Mg}_{0.01}\right){ }^{T(1-3)} \mathrm{Si}_{3.07} \mathrm{O}_{12}(\mathrm{OH})$, corresponds to the end-member formula $\mathrm{MnCeAl}_{2} \mathrm{Mn}^{2+}\left(\mathrm{Si}_{2} \mathrm{O}_{7}\right)\left(\mathrm{SiO}_{4}\right) \mathrm{O}(\mathrm{OH})$, i.e., "androsite-(Ce)". This phase has not been approved yet as a valid mineral species. Both analyses collected on the larger grain of sample \#19903-1 have $\mathrm{Si}>3$ atoms per formula unit (apfu). According to Armbruster et al. [1], if Si becomes $>3.05$ apfu, the formula may be renormalized on $\mathrm{Si}=3 \mathrm{apfu}$. However, although the analysis of the light grey domain has $\mathrm{Si}=3.07 \mathrm{apfu}$, we did prefer to maintain the same kind of normalization used for the other domains.

Sample \#19903-2 is more complex. The core (light grey in Figure 3) has chemical composition ${ }^{A(1)}\left(\mathrm{Mn}^{2+}{ }_{0.53} \mathrm{Ca}_{0.47}\right){ }^{A(2)}\left(\mathrm{Ce}_{0.50} \mathrm{La}_{0.33} \mathrm{Pr}_{0.05} \mathrm{Nd}_{0.05} \mathrm{Ca}_{0.07}\right)^{M(1)}\left(\mathrm{Mn}^{3+}{ }_{0.62} \mathrm{Fe}^{3+}{ }_{0.23} \mathrm{Al}_{0.15}\right)^{M(2)} \mathrm{Al}_{1.00}$ ${ }^{M(3)}\left(\mathrm{Mn}^{2+}{ }_{0.88} \mathrm{Mn}^{3+}{ }_{0.10} \mathrm{Mg}_{0.02}\right)^{T(1-3)}\left(\mathrm{Si}_{2.97} \mathrm{Al}_{0.03}\right) \mathrm{O}_{12}(\mathrm{OH})$. It corresponds to manganiandrosite-(Ce) [10], having the end-member formula $\mathrm{MnCeMn}^{3+} \mathrm{AlMn}^{2+}\left(\mathrm{Si}_{2} \mathrm{O}_{7}\right)\left(\mathrm{SiO}_{4}\right) \mathrm{O}(\mathrm{OH})$. The grey domain corresponds to ferriakasakaite-(Ce), with chemical formula ${ }^{A(1)}\left(\mathrm{Ca}_{0.54} \mathrm{Mn}^{2+}{ }_{0.46}\right){ }^{A(2)}\left(\mathrm{Ce}_{0.35} \mathrm{La}_{0.17} \mathrm{Nd}_{0.07} \mathrm{Pr}_{0.03} \mathrm{Ca}_{0.36} \square_{0.02}\right)$ ${ }^{M(1)}\left(\mathrm{Fe}^{3+}{ }_{0.58} \mathrm{Al}_{0.42}\right) \quad{ }^{M(2)} \mathrm{Al}_{1.00}{ }^{M(3)}\left(\mathrm{Mn}^{2+}{ }_{0.65} \mathrm{Mn}^{3+}{ }_{0.25} \mathrm{Fe}^{3+}{ }_{0.09} \mathrm{Mg}_{0.01}\right){ }^{T(1-3)} \mathrm{Si}_{3.02} \mathrm{O}_{12} \quad(\mathrm{OH})$. Finally, the dark grey domain of the sample \#19903-2 has chemical formula ${ }^{A(1)}\left(\mathrm{Ca}_{0.56} \mathrm{Mn}^{2+}{ }_{0.44}\right)$ ${ }^{A(2)}\left(\mathrm{Ca}_{0.49} \mathrm{Ce}_{0.26} \mathrm{La}_{0.13} \mathrm{Nd}_{0.05} \mathrm{Pr}_{0.02} \square_{0.05}\right)^{M(1)}\left(\mathrm{Al}_{0.57} \mathrm{Fe}^{3+}{ }_{0.43}{ }^{M(2)} \mathrm{Al}_{1.00}{ }^{M(3)}\left(\mathrm{Mn}^{2+}{ }_{0.55} \mathrm{Fe}^{3+}{ }_{0.41} \mathrm{Mn}^{3+}{ }_{0.03} \mathrm{Mg}_{0.01}\right)\right.$ ${ }^{T(1-3)} \mathrm{Si}_{3.04} \mathrm{O}_{12}(\mathrm{OH})$. In this case, it is difficult to propose an end-member formula. Indeed, taking into account the dominant constituents at the $A$ and $M$ sites, the unbalanced chemical formula $\mathrm{CaCaAlAlMn}{ }^{2+}\left(\mathrm{Si}_{2} \mathrm{O}_{7}\right)\left(\mathrm{SiO}_{4}\right) \mathrm{O}(\mathrm{OH})$ is obtained. Likely, the quality of this set of analyses is not satisfying, as proved by the low analytical total (Table 2). However, following [1], we could consider this as a border-line case where $R E E<0.5$, close to $0.5 \mathrm{apfu}$ (i.e., $0.44 \mathrm{apfu}$ ). In this case, the mineral should be assigned to the clinozoisite group (REE $<0.5$ apfu) and the species name should be determined by the dominant $\mathrm{M}^{3+}$ at $M(3)$, i.e., $\mathrm{Fe}^{3+}$. Consequently, this domain could be classified as epidote.

\subsection{X-ray Crystallography}

Powder X-ray diffraction data of manganiakasakaite-(La) and ferriakasakaite-(Ce), collected using a $114.6 \mathrm{~mm}$ Gandolfi camera and $\mathrm{CuK} \alpha$ radiation, are listed in Table 3.

Single-crystal X-ray diffraction data were collected using a Bruker Smart Breeze single-crystal diffractometer with an air-cooled CCD area detector, and graphite-monochromatized MoK $\alpha$ radiation. The detector-to-crystal distance was set at $50 \mathrm{~mm}$. Intensity data were collected using $\omega$ and $\varphi$ scan modes, in $0.5^{\circ}$ slices, with an exposure time of $10 \mathrm{~s}$ per frame. The data were corrected for Lorentz and polarization factors and absorption using the software package Apex2 [12] for manganiakasakaite-(La) and Apex3 [13] for ferriakasakaite-(Ce).

The statistical tests on the distribution of $|E|$ values and the systematic absences confirmed the space group symmetry $P 2_{1} / m$. The crystal structures of both minerals were refined using Shelxl-2018 [14] starting from the atomic coordinates of ferriakasakaite-(La) given by Nagashima et al. [2]. The position of the $\mathrm{H}$ atom was located through difference-Fourier syntheses. The following scattering curves for neutral atoms, taken from the International Tables for Crystallography [15], were used:

- in manganiakasakaite-(La), Ca vs. Mn at $A(1), \mathrm{La}$ vs. Ca at $A(2), \mathrm{Mn}$ vs. $\mathrm{Al}$ at $M(1)$ and $M(3), \mathrm{Al}$ at $M(2)$;

- $\quad$ in ferriakasakaite-(Ce), Ca vs. Mn at $A(1), \mathrm{Ce}$ vs. Ca at $A(2), \mathrm{Fe}$ vs. $\mathrm{Al}$ at $M(1), \mathrm{Al}$ at $M(2), \mathrm{Mn}$ at $M(3)$.

Site occupancies at $\mathrm{Si}(1)-\mathrm{Si}(3)$ sites, $\mathrm{O}(1)-\mathrm{O}(10)$ sites, and $\mathrm{H}$ site were refined using scattering curves of $\mathrm{Si}, \mathrm{O}$, and $\mathrm{H}$, respectively, assuming the full occupancy of these positions. 
Table 3. Observed and calculated X-ray powder diffraction data for manganiakasakaite-(La) and ferriakasakaite-(Ce).

\begin{tabular}{|c|c|c|c|c|c|c|c|c|c|}
\hline \multicolumn{5}{|c|}{ Manganiakasakaite-(La) } & \multicolumn{5}{|c|}{ Ferriakasakaite-(Ce) } \\
\hline$I_{\text {obs }}$ & $d_{\mathrm{obs}}$ & $I_{\text {calc }}$ & $d_{\text {calc }}$ & $h k l$ & $I_{\text {obs }}$ & $d_{\mathrm{obs}}$ & $I_{\text {calc }}$ & $d_{\text {calc }}$ & $h k l$ \\
\hline- & - & 25 & 7.89 & -101 & - & - & 18 & 7.91 & -101 \\
\hline- & - & 14 & 5.17 & 101 & - & - & - & - & - \\
\hline- & - & 14 & 4.976 & -102 & - & - & 15 & 4.987 & -102 \\
\hline- & - & 13 & 4.688 & 110 & mw & 4.65 & 11 & 4.671 & 110 \\
\hline $\mathbf{m}$ & 3.516 & 42 & 3.514 & -211 & $\mathbf{m}$ & 3.519 & 44 & 3.510 & -211 \\
\hline- & - & - & - & - & vw & 3.255 & 10 & 3.260 & 201 \\
\hline \multirow{5}{*}{$\mathbf{s}$} & & 21 & 2.910 & -302 & \multirow[b]{2}{*}{ vs } & \multirow[b]{2}{*}{2.911} & 29 & 2.914 & -302 \\
\hline & & 100 & 2.905 & -113 & & & 100 & 2.904 & -113 \\
\hline & 2.899 & 36 & 2.865 & 020 & \multirow{3}{*}{$\mathbf{m w}$} & \multirow{3}{*}{2.841} & 37 & 2.854 & 020 \\
\hline & & 11 & 2.844 & 211 & & & 11 & 2.831 & 211 \\
\hline & & 14 & 2.718 & 300 & & & 14 & 2.709 & 300 \\
\hline \multirow[t]{2}{*}{$\mathrm{ms}$} & 2.711 & 30 & 2.717 & 013 & \multirow[t]{2}{*}{$\mathrm{ms}$} & \multirow[t]{2}{*}{2.711} & 31 & 2.710 & 013 \\
\hline & & 35 & 2.703 & 120 & & & 31 & 2.693 & 120 \\
\hline \multirow{2}{*}{$\mathbf{m}$} & \multirow[b]{2}{*}{2.621} & 54 & 2.621 & -311 & ms & 2.616 & 51 & 2.617 & -311 \\
\hline & & 28 & 2.587 & 202 & $\mathrm{w}$ & 2.576 & 25 & 2.573 & 202 \\
\hline- & - & - & - & - & vw & 2.508 & 5 & 25.08 & -104 \\
\hline $\mathrm{w}$ & 2.387 & 14 & 2.391 & -313 & mw & 2.404 & 16 & 2.395 & -313 \\
\hline $\mathrm{w}$ & 2.324 & 14 & 2.318 & -222 & $\mathrm{w}$ & 2.294 & 12 & 2.315 & -222 \\
\hline $\mathrm{mw}$ & 2.179 & 22 & 2.184 & -401 & mw & 2.181 & 21 & 2.180 & -401 \\
\hline $\mathrm{mw}$ & 2.165 & 19 & 2.157 & 221 & $\mathrm{mw}$ & 2.144 & 19 & 2.148 & 221 \\
\hline \multirow[b]{2}{*}{$\mathbf{m}$} & \multirow[b]{2}{*}{2.109} & 17 & 2.117 & -223 & w & 2.109 & 17 & 2.116 & -223 \\
\hline & & 16 & 2.100 & 023 & $w$ & 2.082 & 14 & 2.094 & 023 \\
\hline- & - & - & - & - & vw & 2.024 & 2 & 2.022 & -205 \\
\hline $\mathrm{mw}$ & 1.925 & 13 & 1.920 & 222 & \multirow[b]{2}{*}{$\mathrm{mw}$} & \multirow{2}{*}{1.907} & 13 & 1.911 & 222 \\
\hline $\mathrm{w}$ & 1.874 & 11 & 1.878 & -224 & & & 11 & 1.878 & -224 \\
\hline- & - & - & - & - & $\mathrm{vW}$ & 1.782 & 5 & 1.778 & -502 \\
\hline- & - & - & - & - & vw & 1.698 & 6 & 1.696 & -415 \\
\hline \multirow{2}{*}{$\mathbf{m}$} & \multirow[b]{2}{*}{1.665} & 12 & 1.662 & -133 & \multirow{2}{*}{$\mathrm{mw}$} & \multirow[b]{2}{*}{1.644} & 12 & 1.657 & -133 \\
\hline & & 10 & 1.642 & -106 & & & 10 & 1.641 & -106 \\
\hline $\mathrm{mw}$ & 1.628 & 22 & 1.625 & -424 & $\mathrm{mw}$ & 1.628 & 19 & 1.626 & -424 \\
\hline $\mathrm{w}$ & 1.608 & 12 & 1.603 & -331 & $\mathrm{mw}$ & 1.600 & 11 & 1.598 & -331 \\
\hline
\end{tabular}

Note: Intensities and $d_{\mathrm{hkl}}$ were calculated using the software PowderCell 2.3 [11] on the basis of the structural models given in Table 5. Only the reflections with $I_{\text {calc }}>10$ are given, if not observed. The eight strongest reflections are highlighted in bold. Intensities were visually estimated: $\mathrm{vs}=$ very strong; $\mathrm{s}=$ strong; $\mathrm{ms}=$ medium-strong; $\mathrm{m}=$ medium; $\mathrm{mw}$ = medium-weak; $\mathrm{w}$ = weak; vw = very weak.

After several cycles of refinement, an anisotropic structural model (only $\mathrm{H}$ atoms were refined isotropically) converged to 0.0262 for 2119 reflections with $F_{\mathrm{o}}>4 \sigma\left(F_{\mathrm{o}}\right)$ for manganiakasakaite-(La), and to 0.0196 for 1960 reflections with $F_{\mathrm{o}}>4 \sigma\left(F_{\mathrm{o}}\right)$ for ferriakasakaite-(Ce). Details of data collection and refinement are given in Table 4.

Table 4. Crystal data and experimental details for manganiakasakaite-(La) and ferriakasakaite-(Ce).

\begin{tabular}{ccc}
\hline Crystal Data & Manganiakasakaite-(La) & Ferriakasakaite-(Ce) \\
\hline Crystal size $(\mathrm{mm})$ & $0.11 \times 0.08 \times 0.05$ & $0.07 \times 0.05 \times 0.04$ \\
Crystal system, space group & Monoclinic, $P 2_{1} / m$ & Monoclinic, $P 2_{1} / m$ \\
$a(\AA)$ & $8.9057(10)$ & $8.9033(3)$ \\
$b(\AA)$ & $5.7294(6)$ & $5.7066(2)$ \\
$c(\AA)$ & $10.1134(11)$ & $10.1363(3)$ \\
$\beta\left({ }^{\circ}\right)$ & $113.713(5)$ & $114.222(2)$ \\
$V\left(\AA^{3}\right)$ & $472.46(9)$ & $469.66(3)$ \\
$Z$ & 2 & 2 \\
\hline
\end{tabular}


Table 4. Cont.

\begin{tabular}{|c|c|c|}
\hline Crystal Data & Manganiakasakaite-(La) & Ferriakasakaite-(Ce) \\
\hline \multicolumn{3}{|c|}{ Data Collection and Refinement } \\
\hline Radiation, wavelength $(\AA)$ & $\operatorname{MoK} \alpha, 0.71073$ & $\operatorname{MoK} \alpha, 0.71073$ \\
\hline Temperature $(\mathrm{K})$ & 293 & 293 \\
\hline $2 \theta_{\max }\left({ }^{\circ}\right)$ & 70.20 & 70.00 \\
\hline Measured reflections & 8149 & 8636 \\
\hline Unique reflections & 2213 & 2149 \\
\hline Reflections with $F_{\mathrm{o}}>4 \sigma\left(F_{\mathrm{o}}\right)$ & 2119 & 1960 \\
\hline$R_{\text {int }}$ & 0.0188 & 0.0174 \\
\hline \multirow[t]{2}{*}{$R \sigma$} & 0.0185 & 0.0164 \\
\hline & $-14 \leq h \leq 14$ & $-13 \leq h \leq 14$ \\
\hline \multirow[t]{2}{*}{ Range of $h, k, l$} & $-9 \leq k \leq 9$ & $-9 \leq k \leq 9$ \\
\hline & $-15 \leq l \leq 16$ & $-16 \leq l \leq 16$ \\
\hline$R\left[F_{\mathrm{o}}>4 \sigma\left(F_{\mathrm{o}}\right)\right]$ & 0.0262 & 0.0196 \\
\hline$R$ (all data) & 0.0282 & 0.0233 \\
\hline$w R\left(\right.$ on $\left.F_{\mathrm{o}}{ }^{2}\right)$ & 0.0541 & 0.0470 \\
\hline Goof & 1.312 & 1.184 \\
\hline Number of l.s. parameters & 125 & 124 \\
\hline Maximum and minimum residual peak $\left(e \AA^{-3}\right)$ & $\begin{array}{l}1.10 \text { [at } 0.68 \AA \text { from } M(3)] \\
-0.90[\text { at } 0.54 \AA \text { from } \mathrm{Si}(2)]\end{array}$ & $\begin{array}{c}0.84[\text { at } 0.71 \AA \text { from } A(2)] \\
-0.53[\text { at } 0.80 \AA \text { from } \operatorname{Si}(2)]\end{array}$ \\
\hline
\end{tabular}

Fractional atomic coordinates, site occupation factors, and displacement parameters are given in Table 5.

Table 5. Fractional atomic coordinates, site occupation factors (s.o.f.) and isotropic $\left(^{*}\right)$ or equivalent isotropic displacement parameters $\left(\AA^{2}\right)$ for manganiakasakaite-(La) and ferriakasakaite-(Ce).

\begin{tabular}{|c|c|c|c|c|c|}
\hline \multicolumn{6}{|c|}{ Manganiakasakaite-(La) } \\
\hline Site & s.o.f. & $x / a$ & $y / b$ & $z / c$ & $U_{\text {eq }} / U_{\text {iso }}$ \\
\hline$A(1)$ & $\mathrm{Ca}_{0.62(1)} \mathrm{Mn}_{0.38(1)}$ & $0.76148(8)$ & $3 / 4$ & $0.15417(6)$ & $0.01419(15)$ \\
\hline$A(2)$ & $\mathrm{La}_{0.71(1)} \mathrm{Ca}_{0.29(1)}$ & $0.59190(3)$ & $3 / 4$ & $0.42774(2)$ & $0.01191(6)$ \\
\hline$M(1)$ & $\mathrm{Mn}_{0.73(1)} \mathrm{Al}_{0.27(1)}$ & 0 & 0 & 0 & $0.00818(14)$ \\
\hline$M(2)$ & $\mathrm{Al}_{1.00}$ & 0 & 0 & $1 / 2$ & $0.00730(16)$ \\
\hline$M(3)$ & $\mathrm{Mn}_{0.97(1)} \mathrm{Al}_{0.03(1)}$ & $0.31252(7)$ & $1 / 4$ & $0.20956(6)$ & $0.01420(15)$ \\
\hline $\operatorname{Si}(1)$ & $\mathrm{Si}_{1.00}$ & $0.34904(11)$ & $3 / 4$ & $0.03559(9)$ & $0.00923(16)$ \\
\hline $\operatorname{Si}(2)$ & $\mathrm{Si}_{1.00}$ & $0.69314(11)$ & $1 / 4$ & $0.28238(10)$ & $0.01001(16)$ \\
\hline $\operatorname{Si}(3)$ & $\mathrm{Si}_{1.00}$ & $0.19008(10)$ & $3 / 4$ & $0.32345(9)$ & $0.00823(15)$ \\
\hline $\mathrm{O}(1)$ & $\mathrm{O}_{1.00}$ & $0.2441(2)$ & $0.9915(3)$ & $0.0257(2)$ & $0.0161(3)$ \\
\hline $\mathrm{O}(2)$ & $\mathrm{O}_{1.00}$ & $0.3127(2)$ & $0.9745(3)$ & $0.35929(17)$ & $0.0124(3)$ \\
\hline $\mathrm{O}(3)$ & $\mathrm{O}_{1.00}$ & $0.8037(2)$ & $0.0137(3)$ & $0.33266(19)$ & $0.0142(3)$ \\
\hline $\mathrm{O}(4)$ & $\mathrm{O}_{1.00}$ & $0.0565(3)$ & $1 / 4$ & $0.1321(2)$ & $0.0118(4)$ \\
\hline $\mathrm{O}(5)$ & $\mathrm{O}_{1.00}$ & $0.0482(3)$ & $3 / 4$ & $0.1565(3)$ & $0.0132(4)$ \\
\hline $\mathrm{O}(6)$ & $\mathrm{O}_{1.00}$ & $0.0777(3)$ & $3 / 4$ & $0.4190(3)$ & $0.0119(4)$ \\
\hline $\mathrm{O}(7)$ & $\mathrm{O}_{1.00}$ & $0.5165(3)$ & $3 / 4$ & $0.1764(3)$ & $0.0163(4)$ \\
\hline $\mathrm{O}(8)$ & $\mathrm{O}_{1.00}$ & $0.5542(4)$ & $1 / 4$ & $0.3439(4)$ & $0.0242(6)$ \\
\hline $\mathrm{O}(9)$ & $\mathrm{O}_{1.00}$ & $0.6052(4)$ & $1 / 4$ & $0.1056(3)$ & $0.0196(5)$ \\
\hline $\mathrm{O}(10)$ & $\mathrm{O}_{1.00}$ & $0.0909(3)$ & $1 / 4$ & $0.4329(3)$ & $0.0112(4)$ \\
\hline $\mathrm{H}(10)$ & $\mathrm{H}_{1.00}$ & $0.072(7)$ & $1 / 4$ & $0.345(6)$ & $0.021(13)$ * \\
\hline \multicolumn{6}{|c|}{ Ferriakasakaite-(Ce) } \\
\hline Site & s.o.f. & $x / a$ & $y / b$ & $z / c$ & $U_{\mathrm{eq}} / U_{\text {iso }}$ \\
\hline$A(1)$ & $\mathrm{Ca}_{0.67(1)} \mathrm{Mn}_{0.33(1)}$ & $0.75979(5)$ & $3 / 4$ & $0.15278(4)$ & $0.01261(11)$ \\
\hline$A(2)$ & $\mathrm{Ce}_{0.62(1)} \mathrm{Ca}_{0.38(1)}$ & $0.59315(2)$ & $3 / 4$ & $0.42650(2)$ & $0.01213(6)$ \\
\hline$M(1)$ & $\mathrm{Fe}_{0.54(1)} \mathrm{Al}_{0.46(1)}$ & 0 & 0 & 0 & $0.00761(12)$ \\
\hline$M(2)$ & $\mathrm{Al}_{1.00}$ & 0 & 0 & $1 / 2$ & $0.00718(12)$ \\
\hline$M(3)$ & $\mathrm{Mn}_{1.00}$ & $0.30614(4)$ & $1 / 4$ & $0.21358(4)$ & $0.01268(9)$ \\
\hline
\end{tabular}


Table 5. Cont.

\begin{tabular}{cccccc}
\hline \multicolumn{5}{c}{ Ferriakasakaite-(Ce) } \\
\hline Site & s.o.f. & $x / a$ & $y / b$ & $z / c$ & $U_{\text {eq }} / \boldsymbol{U}_{\text {iso }}$ \\
\hline $\mathrm{Si}(1)$ & $\mathrm{Si}_{1.00}$ & $0.34584(8)$ & $3 / 4$ & $0.03898(7)$ & $0.00937(12)$ \\
$\mathrm{Si}(2)$ & $\mathrm{Si}_{1.00}$ & $0.69169(7)$ & $1 / 4$ & $0.27991(6)$ & $0.00865(11)$ \\
$\mathrm{Si}(3)$ & $\mathrm{Si}_{1.00}$ & $0.18835(7)$ & $3 / 4$ & $0.32267(6)$ & $0.00784(11)$ \\
$\mathrm{O}(1)$ & $\mathrm{O}_{1.00}$ & $0.24201(15)$ & $0.9919(2)$ & $0.03085(15)$ & $0.0163(2)$ \\
$\mathrm{O}(2)$ & $\mathrm{O}_{1.00}$ & $0.31067(14)$ & $0.9753(2)$ & $0.35912(12)$ & $0.0122(2)$ \\
$\mathrm{O}(3)$ & $\mathrm{O}_{1.00}$ & $0.80235(15)$ & $0.0137(2)$ & $0.33378(13)$ & $0.0138(2)$ \\
$\mathrm{O}(4)$ & $\mathrm{O}_{1.00}$ & $0.0548(2)$ & $1 / 4$ & $0.13134(18)$ & $0.0114(3)$ \\
$\mathrm{O}(5)$ & $\mathrm{O}_{1.00}$ & $0.0457(2)$ & $3 / 4$ & $0.15434(17)$ & $0.0119(3)$ \\
$\mathrm{O}(6)$ & $\mathrm{O}_{1.00}$ & $0.0756(2)$ & $3 / 4$ & $0.41694(18)$ & $0.0104(3)$ \\
$\mathrm{O}(7)$ & $\mathrm{O}_{1.00}$ & $0.5158(2)$ & $3 / 4$ & $0.17657(19)$ & $0.0166(3)$ \\
$\mathrm{O}(8)$ & $\mathrm{O}_{1.00}$ & $0.5468(2)$ & $1 / 4$ & $0.3348(2)$ & $0.0232(4)$ \\
$\mathrm{O}(9)$ & $\mathrm{O}_{1.00}$ & $0.6118(2)$ & $1 / 4$ & $0.10326(19)$ & $0.0204(4)$ \\
$\mathrm{O}(10)$ & $\mathrm{O}_{1.00}$ & $0.0889(2)$ & $1 / 4$ & $0.43213(18)$ & $0.0104(3)$ \\
$\mathrm{H}(10)$ & $\mathrm{H}_{1.00}$ & $0.063(5)$ & $1 / 4$ & $0.343(5)$ & $0.039(12)$ \\
\hline
\end{tabular}

Table 6 reports selected bond distances, whereas Table 7 shows the comparison between observed site scattering and that calculated on the basis of the proposed site population.

Table 6. Selected bond distances $(\AA)$ for manganiakasakaite-(La) [Mn-ak-(La)] and ferriakasakaite-(Ce) [Fe-ak-(Ce)].

\begin{tabular}{|c|c|c|c|c|c|c|c|c|}
\hline & Mn-ak-(La) & Fe-ak-(Ce) & & Mn-ak-(La) & Fe-ak-(Ce) & & Mn-ak-(La) & Fe-ak-(Ce) \\
\hline$A(1)-\mathrm{O}(3) \times 2$ & $2.2663(18)$ & 2.2814(12) & $M(1)-\mathrm{O}(4) \times 2$ & $1.8841(15)$ & $1.8742(11)$ & $\mathrm{Si}(1)-\mathrm{O}(7)$ & $1.595(3)$ & $1.5818(18)$ \\
\hline$A(1)-\mathrm{O}(7)$ & $2.281(3)$ & $2.282(2)$ & $M(1)-\mathrm{O}(5) \times 2$ & 2.0481(17) & $2.0313(11)$ & $\mathrm{Si}(1)-\mathrm{O}(9)$ & $1.634(3)$ & $1.6329(19)$ \\
\hline$A(1)-\mathrm{O}(1) \times 2$ & $2.3304(19)$ & $2.3684(14)$ & $M(1)-\mathrm{O}(1) \times 2$ & $2.0835(18)$ & $2.0469(13)$ & $\mathrm{Si}(1)-\mathrm{O}(1) \times 2$ & $1.6503(18)$ & $1.6445(13)$ \\
\hline$A(1)-\mathrm{O}(5)$ & $2.544(2)$ & $2.5387(18)$ & $\langle M(1)-\mathrm{O}\rangle$ & 2.005 & 1.984 & $<\mathrm{Si}(1)-\mathrm{O}\rangle$ & 1.632 & 1.626 \\
\hline$A(1)-\mathrm{O}(6)$ & $3.006(2)$ & $2.9828(17)$ & & & & & & \\
\hline \multirow[t]{2}{*}{$<A 1-\mathrm{O}>$} & 2.432 & 2.443 & $M(2)-\mathrm{O}(3) \times 2$ & $1.8832(17)$ & $1.8732(12)$ & $\mathrm{Si}(2)-\mathrm{O}(8)$ & $1.593(3)$ & $1.6002(19)$ \\
\hline & & & $M(2)-\mathrm{O}(10) \times 2$ & $1.8999(15)$ & $1.8928(11)$ & $\mathrm{Si}(2)-\mathrm{O}(3) \times 2$ & $1.6305(17)$ & $1.6264(12)$ \\
\hline$A(2)-\mathrm{O}(7)$ & $2.354(2)$ & $2.3367(18)$ & $M(2)-\mathrm{O}(6) \times 2$ & $1.9128(15)$ & $1.9136(10)$ & $\mathrm{Si}(2)-\mathrm{O}(9)$ & $1.638(3)$ & 1.6331(19) \\
\hline$A(2)-\mathrm{O}(2) \times 2$ & $2.5259(16)$ & $2.5269(12)$ & $<M(2)-\mathrm{O}>$ & 1.899 & 1.893 & $<\mathrm{Si}(2)-\mathrm{O}>$ & 1.623 & 1.622 \\
\hline$A(2)-\mathrm{O}(10)$ & $2.601(2)$ & 2.5955(17) & & & & & & \\
\hline$A(2)-\mathrm{O}(2) \times 2$ & $2.6329(17)$ & $2.6525(12)$ & $M(3)-\mathrm{O}(8)$ & $2.031(3)$ & $1.985(2)$ & $\mathrm{Si}(3)-\mathrm{O}(2) \times 2$ & $1.6309(17)$ & $1.6266(12)$ \\
\hline$A(2)-\mathrm{O}(3) \times 2$ & $2.8678(18)$ & 2.8379(13) & $M(3)-\mathrm{O}(4)$ & $2.092(2)$ & $2.0427(17)$ & $\mathrm{Si}(3)-\mathrm{O}(6)$ & $1.647(2)$ & $1.6457(17)$ \\
\hline$A(2)-\mathrm{O}(8) \times 2$ & $2.9680(9)$ & $2.9768(6)$ & $M(3)-\mathrm{O}(2) \times 2$ & 2.1869(17) & $2.1415(12)$ & $\mathrm{Si}(3)-\mathrm{O}(5)$ & $1.653(2)$ & 1.6573(17) \\
\hline \multirow[t]{2}{*}{$<A(2)-\mathrm{O}>$} & 2.694 & 2.692 & $M(3)-\mathrm{O}(1) \times 2$ & $2.2607(18)$ & $2.2491(13)$ & $<\mathrm{Si}(3)-\mathrm{O}>$ & 1.640 & 1.639 \\
\hline & & & $\langle M(3)-\mathrm{O}\rangle$ & 2.170 & 2.135 & & & \\
\hline
\end{tabular}

Table 7. Refined site scattering vs. calculated site scattering (in electrons) and site population at the $A$ and $M$ sites in manganiakasakaite-(La) and ferriakasakaite-(Ce).

\begin{tabular}{cccc}
\hline \multicolumn{3}{c}{ Manganiakasakaite-(La) } \\
\hline Site & Refined site scattering & Proposed site population & Calculated site scattering \\
$A(1)$ & 21.9 & $\mathrm{Ca}_{0.61} \mathrm{Mn}_{0.39}$ & 22.0 \\
$A(2)$ & 46.3 & $\mathrm{La}_{0.52} \mathrm{Nd}_{0.08} \mathrm{Ce}_{0.07} \mathrm{Pr}_{0.07} \mathrm{Y}_{0.01} \mathrm{Ca}_{0.25}$ & 48.0 \\
$M(1)$ & 21.8 & $\mathrm{Mn}^{3+}{ }_{0.52} \mathrm{Fe}^{3+}{ }_{0.28} \mathrm{Al}_{0.19} \mathrm{~V}^{3+}{ }_{0.01}$ & 23.0 \\
$M(2)$ & 13.0 & $\mathrm{Al}_{1.00}$ & 13.0 \\
$M(3)$ & 24.6 & $\mathrm{Mn}^{2+}{ }_{0.60} \mathrm{Mn}^{3+}{ }_{0.27} \mathrm{Mg}_{0.13}$ & 23.3 \\
\hline & & Ferriakasakaite-(Ce) & \\
\hline Site & Refined site scattering & Proposed site population & Calculated site scattering \\
$A(1)$ & 21.6 & $\mathrm{Ca}_{0.60} \mathrm{Mn}_{0.40}$ & 22.0 \\
$A(2)$ & 43.6 & $\mathrm{Ce}_{0.37} \mathrm{La}_{0.17} \mathrm{Nd}_{0.06} \mathrm{Ca}$ & 42.8 \\
$M(1)$ & 20.0 & $\mathrm{Fe}^{3+}{ }_{0.60} \mathrm{Al}_{0.40}$ & 20.8 \\
$M(2)$ & 13.0 & $\mathrm{Al}_{1.00}$ & 13.0 \\
$M(3)$ & 25.0 & $\mathrm{Mn}^{2+}{ }_{0.65} \mathrm{Mn}^{3+}{ }_{0.35}$ & 25.0 \\
\hline
\end{tabular}


Finally, Table 8 gives the bond-valence calculations obtained using the bond-valence parameters of Brese and $\mathrm{O}^{\prime}$ Keeffe [16]. The oxygen atom hosted at the $\mathrm{O}(10)$ site is underbonded, being involved in $\mathrm{H}$-bond. Indeed, it represents an $\mathrm{OH}$ group, with $\mathrm{O}(10)-\mathrm{H}(10)$ distance of $0.84(6)$ and $0.84(5) \AA$ in manganiakasakaite-(La) and ferriakasakaite-(Ce), respectively. The acceptor is $\mathrm{O}(4)$, with a $\mathrm{O}(10) \cdots \mathrm{O}(4)$ distance of 2.932(3) $\AA$ in the former and 2.937(2) $\AA$ in the latter. Finally, the $\mathrm{O}(10)-\mathrm{H}(10) \cdots \mathrm{O}(4)$ angle is $173(5)^{\circ}$ in manganiakasakaite-(La) and $167(4)^{\circ}$ in ferriakasakaiate-(Ce).

Table 8. Weighted bond-valence balance (in v.u.) in manganiakasakaite-(La) and ferriakasakaite-(Ce).

\begin{tabular}{|c|c|c|c|c|c|c|c|c|c|}
\hline \multicolumn{10}{|c|}{ Manganiakasakaite-(La) } \\
\hline & $A(1)$ & $A(2)$ & $M(1)$ & $M(2)$ & $M(3)$ & $\operatorname{Si}(1)$ & $\operatorname{Si}(2)$ & $\operatorname{Si}(3)$ & $\Sigma_{\mathrm{a}} v$ \\
\hline $\mathbf{O}(\mathbf{1})$ & $0.32^{\downarrow \times 2}$ & & $0.40^{\downarrow \times 2}$ & & $0.27^{\downarrow \times 2}$ & $0.93^{\downarrow \times 2}$ & & & 1.92 \\
\hline $\mathrm{O}(2)$ & & $\begin{array}{l}0.33^{\downarrow \times 2} \\
0.25^{\downarrow \times 2}\end{array}$ & & & $0.32^{\downarrow \times 2}$ & & & $0.98^{\downarrow \times 2}$ & 1.88 \\
\hline $\mathrm{O}(3)$ & $0.38^{\downarrow \times 2}$ & $0.13^{\downarrow \times 2}$ & & $0.53^{\downarrow \times 2}$ & & & $0.98^{\downarrow \times 2}$ & & 2.02 \\
\hline $\mathrm{O}(4)$ & & & $2 x \rightarrow 0.68^{\downarrow \times 2}$ & & 0.42 & & & & $1.92^{\mathrm{a}}$ \\
\hline $\mathrm{O}(5)$ & 0.18 & & $2 x \rightarrow 0.44^{\downarrow \times 2}$ & & & & & 0.92 & 1.98 \\
\hline $\mathrm{O}(6)$ & 0.05 & & & $2 x \rightarrow 0.49^{\downarrow \times 2}$ & & & & 0.94 & 1.97 \\
\hline $\mathrm{O}(7)$ & 0.36 & 0.53 & & & & 1.08 & & & 1.97 \\
\hline $\mathrm{O}(8)$ & & $2 x \rightarrow 0.10^{\downarrow \times 2}$ & & & 0.49 & & 1.09 & & 1.78 \\
\hline $\mathrm{O}(9)$ & & & & & & 0.97 & 0.96 & & 1.93 \\
\hline $\mathrm{O}(10)$ & & 0.27 & & $2 x \rightarrow 0.51^{\downarrow \times 2}$ & & & & & $1.15^{b}$ \\
\hline$\Sigma_{\mathrm{c}} v$ & 1.99 & 2.42 & 3.04 & 3.06 & 2.09 & 3.91 & 4.01 & 3.82 & \\
\hline Theor. & 2.00 & 2.75 & 3.00 & 3.00 & 2.27 & 4.00 & 4.00 & 4.00 & \\
\hline \multicolumn{10}{|c|}{ Ferriakasakaite-(Ce) } \\
\hline & $A(1)$ & $A(2)$ & $M(1)$ & $M(2)$ & $M(3)$ & $\operatorname{Si(1)}$ & $\mathrm{Si}(2)$ & $\mathrm{Si}(3)$ & $\Sigma_{\mathbf{a}} v$ \\
\hline $\mathbf{O}(1)$ & $0.29^{\downarrow \times 2}$ & & $0.41^{\downarrow \times 2}$ & & $0.28^{\downarrow \times 2}$ & $0.95^{\downarrow \times 2}$ & & & 1.93 \\
\hline $\mathrm{O}(2)$ & & $\begin{array}{l}0.31^{\downarrow \times 2} \\
0.22^{\downarrow \times 2}\end{array}$ & & & $0.38^{\downarrow \times 2}$ & & & $0.99 \downarrow \times 2$ & 1.90 \\
\hline $\mathrm{O}(3)$ & $0.36^{\downarrow \times 2}$ & $0.13^{\downarrow \times 2}$ & & $0.55^{\downarrow \times 2}$ & & & $0.99^{\downarrow} \times 2$ & & 2.03 \\
\hline $\mathrm{O}(4)$ & & & $2 x \rightarrow 0.66^{\downarrow \times 2}$ & & 0.49 & & & & $1.95^{\mathrm{a}}$ \\
\hline $\mathrm{O}(5)$ & 0.18 & & $2 x \rightarrow 0.43^{\downarrow \times 2}$ & & & & & 0.91 & 1.95 \\
\hline $\mathrm{O}(6)$ & 0.05 & & & $2 x \rightarrow 0.49 \downarrow \times 2$ & & & & 0.94 & 1.97 \\
\hline $\mathrm{O}(7)$ & 0.36 & 0.51 & & & & 1.12 & & & 1.99 \\
\hline $\mathrm{O}(8)$ & & $2 x \rightarrow 0.09 \downarrow \times 2$ & & & 0.57 & & 1.07 & & 1.82 \\
\hline $\mathrm{O}(9)$ & & & & & & 0.98 & 0.98 & & 1.96 \\
\hline $\mathrm{O}(10)$ & & 0.26 & & $2 x \rightarrow 0.52^{\downarrow \times 2}$ & & & & & $1.16^{b}$ \\
\hline$\Sigma_{\mathrm{c}} v$ & 1.89 & 2.27 & 3.00 & 3.12 & 2.38 & 4.00 & 4.03 & 3.83 & \\
\hline Theor. & 2.00 & 2.60 & 3.00 & 3.00 & 2.35 & 4.00 & 4.00 & 4.00 & \\
\hline
\end{tabular}

Note: $\Sigma_{\mathrm{c}} v$ and $\Sigma_{\mathrm{a}} v$ are the bond-valence sums over cations and anions, respectively. Left and right superscripts indicate the number of equivalent bonds for each anion and cation, respectively. For sites with mixed occupancy, the bond valences have been weighted. The contribution of H-bond has been evaluated following Ferraris and Ivaldi [17]. ${ }^{a}$ Includes +0.14 v.u. for the hydrogen bond. ${ }^{b}$ Includes -0.14 v.u. for the hydrogen bond.

The CIF of manganiakasakaite-(La) and ferriakasakaite-(Ce) are available as Supplementary Material linked to this article.

\section{Results and Discussion}

\subsection{Crystal Structure Description}

Manganiakasakaite-(La) and ferriakasakaite-(Ce) are isotypic with the other members of the epidote supergroup, e.g., [18]. Their crystal structure (Figure 4) consists of single chains of edge-sharing $M(2)$-centered octahedra and zig-zag chains of $M(1)$-centered octahedra with $M(3)$-centered octahedra attached on alternate sides along $\mathbf{b} . \mathrm{Si}_{2} \mathrm{O}_{7}$ and $\mathrm{SiO}_{4}$ groups are linked to the octahedral chains. In this way, a framework hosting two types of structural cavities arises. The smaller cavity hosts the $A(1)$ site, whereas the larger one hosts the $A(2)$ site. 


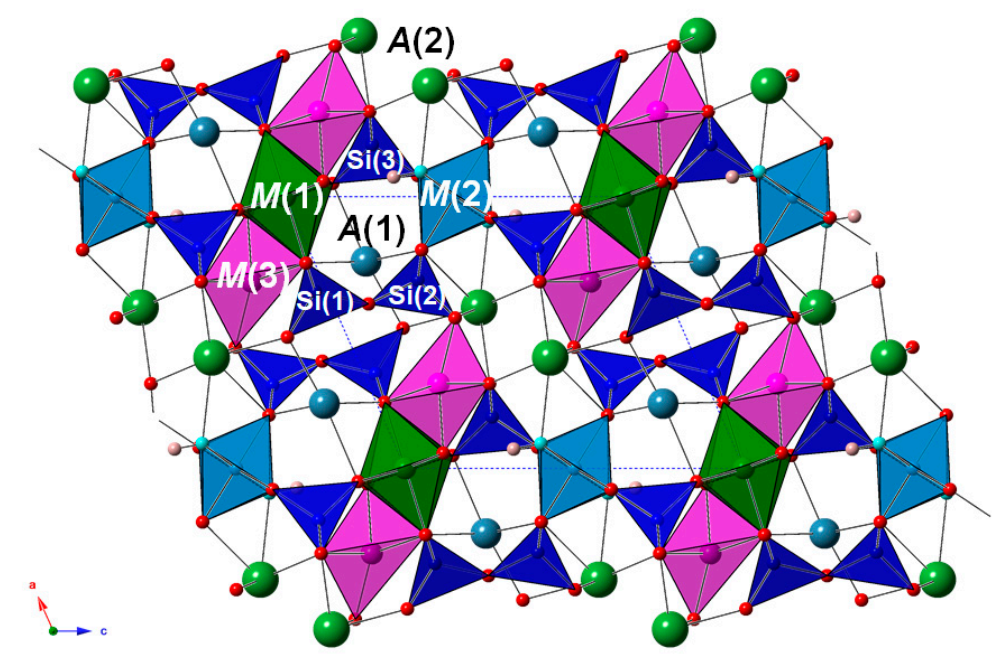

Figure 4. Crystal structure of epidote supergroup minerals as seen down [010].

\subsection{Crystal-Chemistry of Manganiakasakaite-(La) and Ferriakasakaite-(Ce)}

Manganiakasakaite-(La) and ferriakasakaite-(Ce) are characterized by the occurrence of $R E E^{3+}$ at $A(2)$ and $\mathrm{Mn}^{2+}$ at $M(3)$; a trivalent transition metal $\left(\mathrm{Mn}^{3+}\right.$ and $\mathrm{Fe}^{3+}$, respectively) is dominant at $M(1)$.

The $A(1)$ site has a mixed (Ca, Mn) population. The occurrence of $\mathrm{Mn}^{2+}$ replacing $\mathrm{Ca}^{2+}$ agrees with the modified arrangement of the bonded $\mathrm{O}$, with the seventh $\mathrm{O}(6)$ atom shifted away from $A(1)$. In this way, the latter can be appropriately defined as six-fold coordinated. Bonazzi et al. [19] discussed the relation between the difference in bond distances $A(1)-\mathrm{O}(6)$ and $A(1)-\mathrm{O}(5), \mathrm{O}(5)$ being the sixth ligand, and the ${ }^{A(1)} \mathrm{Mn}^{2+}$ content (Figure 5). Such a difference, indicated as $\delta_{7-6}$, is $0.462 \AA$ and $0.444 \AA$ in manganiakasakaite-(La) and ferriakasakaite-(Ce), respectively (purple and blue diamonds in Figure 5, respectively).

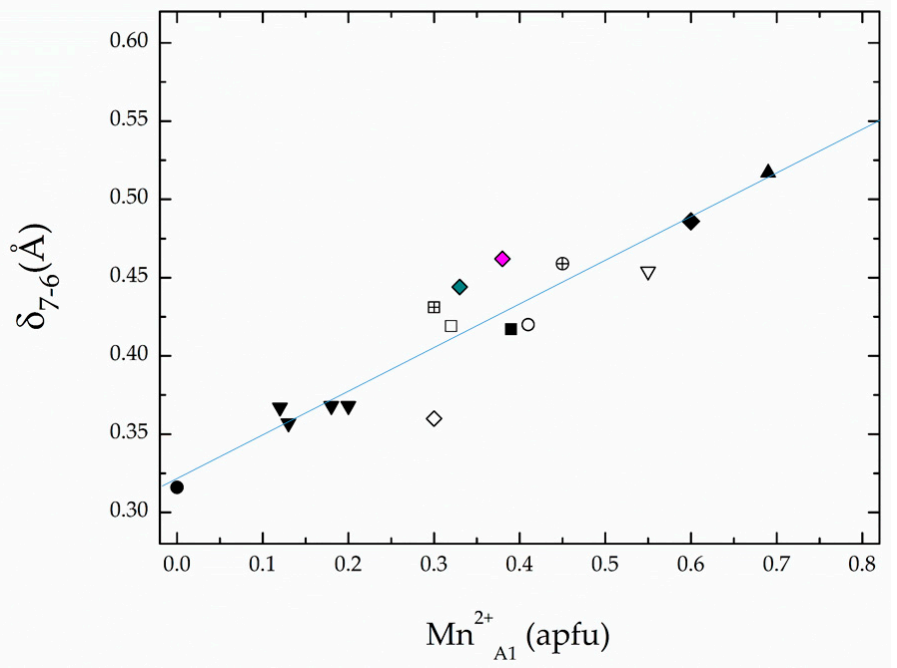

Figure 5. Relation between the $\delta_{7-6}$ value (in $\AA$ ) and the $\mathrm{Mn}^{2+}$ content at the $A(1)$ site. The equation of the regression line is: $\delta_{7-6}=0.32(1)+0.28(3) \mathrm{Mn}^{2+} \mathrm{A} 1\left(r^{2}=0.868\right)$. Symbols refer to data from literature as follows: solid circle $=$ piemontite [20]; solid downward triangle $=R E E$-bearing piemontite [21]; open diamond = ferriakasakaite-(La) [22]; open square = REE-bearing piemontite [19]; crossed open square $=(R E E, \mathrm{~Pb})$-rich piemontite [23]; solid square $=$ vanadoallanite-(La) $[24]$; open circle $=\mathrm{Mn}^{2+}$-rich piemontite [25]; crossed open circle = ferriakasakaite-(La) [2]; open downward triangle = ferriandrosite-(La) [2]; solid diamond = manganiandrosite-(La) [19] and manganiandrosite-(Ce) [10]; solid upward triangle $=$ vanadoandrosite-(Ce) [10]. Blue and purple diamonds refer to ferriakasakaite-(Ce) and manganiakasakaite-(La), respectively (this study). 
The decrease of coordination number is also related to the shift of $\mathrm{O}(9)$ away from the cation at the $A(1)$ site. This shift is associated with a reduction of the $\mathrm{Si}(1)-\mathrm{O}(9)-\mathrm{Si}(2)$ angle, related not only to the site occupancy at $A(1)$ but also to the mean bond length at $M(3)$ [10]. In manganiakasakaite-(La), O(9) is at 3.14 and $3.27 \AA$ from $A(1)$, and the $\mathrm{Si}(1)-\mathrm{O}(9)-\mathrm{Si}(2)$ angle is $140.88(19)^{\circ}$; in ferriakasakaite-(Ce), the same values are 3.09 and $3.26 \AA$, and the angular value is $144.47(14)^{\circ}$.

The $A(2)$ site is a mixed (REE,Ca) site. Lanthanum is the dominant REE in manganiakasakaite-(La), whereas $\mathrm{Ce}$ is dominant in ferriakasakaite-(Ce). Bonazzi et al. [19] discussed the relation between the ratio of $c$ to the unit-cell volume and the REE content in minerals of the piemontite-androsite series. Moreover, they observed a linear relation between the $R E E$ content and the $\beta$ angle. In particular, the latter decreases systematically with increasing $R E E$ and the values observed in manganiakasakaite-(La) $\left(\beta=113.7^{\circ}\right)$ and ferriakasakaite- $(\mathrm{Ce})\left(\beta=114.2^{\circ}\right)$ can be compared with those of other allanite group members, e.g., manganiandrosite-(La) $\left(\beta=113.9^{\circ}[19]\right)$, ferriakasakaite- $(\mathrm{La})(\beta=$ $113.8^{\circ}$ [2] and $\left.114.1^{\circ}[22]\right)$, ferriandrosite-(La) $\left(\beta=113.9^{\circ}[2]\right)$, manganiandrosite- $(\mathrm{Ce})\left(\beta=113.4^{\circ}[10]\right)$, vanadoandrosite-(Ce) $\left(\beta=113.1^{\circ}[10]\right)$, and vanadoallanite-(La) $\left(\beta=114.1^{\circ}\right.$ [24]).

Among the three independent $M$ sites, $M(2)$ is occupied by $\mathrm{Al}$ only. The $<M(2)-\mathrm{O}>$ distances in manganiakasakaite-(La) and ferriakasakaite-(Ce) are 1.899 and $1.893 \AA$, to be compared with values observed in other allanite group minerals, e.g., $1.901 \AA$ in ferriakasakaite-(La) [2] and $1.894 \AA$ in vanadoandrosite-(Ce) [10].

Divalent manganese is located at $M(3)$, in keeping with the average $<M(3)-\mathrm{O}>$ distances, larger than those occurring at $M(1)$, i.e., $2.170 \AA$ vs. $2.005 \AA$ in manganiakasakaite-(La) and $2.135 \AA$ vs. $1.984 \AA$ in ferriakasakaite-(Ce). These values can be compared with the average $<M(3)-\mathrm{O}>$ and $<M(1)-\mathrm{O}>$ distances reported for other allanite group minerals having $\mathrm{Mn}^{2+}$ as dominant M(3) cation, e.g., manganiandrosite-(Ce) (2.197 and 2.019 А [10]), vanadoandrosite-(Ce) (2.195 and $2.011 \AA$ [10]), ferriakasakaite-(La) (2.179 and 2.002 $\AA$ [2]), ferriandrosite-(La) (2.180 and 2.002 $\AA$ [2]), and manganiandrosite-(La) (2.159 and $2.010 \AA$ [19]).

Whereas the assignment of $\mathrm{Mn}^{2+}$ is straightforward, the ordering of $\mathrm{Mn}^{3+}$ and $\mathrm{Fe}^{3+}$ between $\mathrm{M}(1)$ and $M(3)$ is more questionable. Bonazzi and Menchetti [26] reviewed the studies about the ordering of these two transition elements between $M(1)$ and $M(3)$ in natural and synthetic piemontite and pointed out that Mössbauer spectroscopy [27] and neutron diffraction [28] data indicate that not all $\mathrm{Mn}^{3+}$ is ordered at $M(3)$ at the expense of $\mathrm{Fe}^{3+}$. As Langer et al. [29] suggested, although the compression of the $M(3)$ octahedron along the $\mathrm{O}(4)-M(3)-\mathrm{O}(8)$ axis is directly related to the $\mathrm{Mn}^{3+}$ content at this site, there is evidence that the distortion of $\mathrm{M(3)}$ in $\mathrm{Mn}^{3+}$-bearing epidote supergroup minerals is not related to the Jahn-Teller effect only, and that the distortion of the $M$ sites is only partially affected by their composition. Moreover, a significant distortion of the $M(1)$ octahedron along the $\mathrm{O}(4)-\mathrm{M}(1)-\mathrm{O}(4)$ axis seems to be also related with the increasing total $\mathrm{Mn}^{3+}$ content in piemontites [29], thus indicating that part of $\mathrm{Mn}^{3+}$ can be hosted at $\mathrm{M}(1)$. Nonetheless, because the derivation of a mineral name from chemical data should be possible also in the lack of non-conventional structural data (e.g., neutron diffraction), the octahedral cations are assigned to $M(3)$ and $M(1)$ following the procedures suggested by Armbruster et al. [1], i.e., according to a sequence based on decreasing ionic radii, filling first $M(3)$ and then $\mathrm{M}(1)$, and regarding $\mathrm{Mn}^{3+}$ as larger than $\mathrm{Fe}^{3+}$ owing to the Jahn-Teller distortion. Therefore, $\mathrm{Mn}^{3+}$ rather than $\mathrm{Fe}^{3+}$ is assumed to fill first $M(3)$ and then $M(1)$, resulting in the following cation distributions: ${ }^{M(1)}\left(\mathrm{Mn}^{3+}{ }_{0.523} \mathrm{Fe}^{3+}{ }_{0.283} \mathrm{Al}_{0.178} \mathrm{~V}^{3+}{ }_{0.013}\right)$ and ${ }^{M(3)}\left(\mathrm{Mn}^{2+}{ }_{0.603} \mathrm{Mn}^{3+}{ }_{0.269} \mathrm{Mg}_{0.128}\right)$ in manganiakasakaite-(La) and ${ }^{M(1)}\left(\mathrm{Fe}^{3+}{ }_{0.61} \mathrm{Al}_{0.39}\right)$ and ${ }^{M(3)}\left(\mathrm{Mn}^{2+}{ }_{0.64} \mathrm{Mn}^{3+}{ }_{0.33} \mathrm{Fe}^{3+}{ }_{0.02} \mathrm{Mg}_{0.01}\right)$ in ferriakasakaite-(Ce). These site populations agree with the refined site scattering (Table 7) and the related bond-valence sums are in accord with the theoretical value (Table 8).

\subsection{Relationships with other Allanite Group Minerals}

Manganiakasakaite-(La) and ferriakasakaite-(Ce) are two new additions to the allanite group (Table 9), which is currently formed by 17 mineral species. Within the allanite group, species having $\mathrm{Ca}^{2+}$ or $\mathrm{Mn}^{2+}$ as dominant $A(1)$ constituents occur. The presence of about $0.4^{A(1)} \mathrm{Mn}^{2+}$ in the two new 
epidote group minerals from Monte Maniglia, as well as the identification of "androsite-(Ce)" and manganiandrosite-(Ce) in a strongly zoned grains containing ferriakasakaite-(Ce) suggest a continuous substitutional series between ${ }^{A(1)} \mathrm{Ca}$ and ${ }^{A(1)} \mathrm{Mn}^{2+}$ end-member composition and, in particular, between "akasakaites" and "androsites".

Table 9. Members of the allanite group within the epidote supergroup.

\begin{tabular}{ccccccc}
\hline Species & $\boldsymbol{A ( 1 )}$ & $\mathbf{A ( 2 )}$ & $\mathbf{M ( 1 )}$ & $\mathbf{M ( 2 )}$ & $\mathbf{M ( 3 )}$ & Reference \\
\hline Allanite-(Ce) & $\mathrm{Ca}$ & $\mathrm{Ce}$ & $\mathrm{Al}$ & $\mathrm{Al}$ & $\mathrm{Fe}^{2+}$ & Thomson [30] \\
Allanite-(La) & $\mathrm{Ca}$ & $\mathrm{La}$ & $\mathrm{Al}$ & $\mathrm{Al}$ & $\mathrm{Fe}^{2+}$ & Orlandi and Pasero [31] \\
Allanite-(Nd) & $\mathrm{Ca}$ & $\mathrm{Nd}$ & $\mathrm{Al}$ & $\mathrm{Al}$ & $\mathrm{Fe}^{2+}$ & Śkoda et al. [32] \\
Allanite-(Y) & $\mathrm{Ca}$ & $\mathrm{Y}$ & $\mathrm{Al}$ & $\mathrm{Al}$ & $\mathrm{Fe}^{2+}$ & Nel et al. [33] \\
Dissakisite-(Ce) & $\mathrm{Ca}$ & $\mathrm{Ce}$ & $\mathrm{Al}$ & $\mathrm{Al}$ & $\mathrm{Mg}$ & Grew et al. [34] \\
Dissakisite-(La) & $\mathrm{Ca}$ & $\mathrm{La}$ & $\mathrm{Al}$ & $\mathrm{Al}$ & $\mathrm{Mg}$ & Tumiati et al. [35] \\
Ferriakasakaite-(Ce) & $\mathrm{Ca}$ & $\mathrm{Ce}$ & $\mathrm{Fe}^{3+}$ & $\mathrm{Al}$ & $\mathrm{Mn}^{2+}$ & this work \\
Ferriakasakaite-(La) & $\mathrm{Ca}$ & $\mathrm{La}$ & $\mathrm{Fe}^{3+}$ & $\mathrm{Al}$ & $\mathrm{Mn}^{2+}$ & Nagashima et al. [2] \\
Ferriallanite-(Ce) & $\mathrm{Ca}$ & $\mathrm{Ce}$ & $\mathrm{Fe}^{3+}$ & $\mathrm{Al}$ & $\mathrm{Fe}^{2+}$ & Kartashov et al. [36] \\
Ferriallanite-(La) & $\mathrm{Ca}$ & $\mathrm{La}$ & $\mathrm{Fe}^{3+}$ & $\mathrm{Al}$ & $\mathrm{Fe}^{2+}$ & Kolitsch et al. [37] \\
Ferriandrosite-(La) & $\mathrm{Mn}{ }^{2+}$ & $\mathrm{La}$ & $\mathrm{Fe}^{3+}$ & $\mathrm{Al}$ & $\mathrm{Mn}^{2+}$ & Nagashima et al. [2] \\
Manganiakasakaite-(La) & $\mathrm{Ca}$ & $\mathrm{La}$ & $\mathrm{Mn}^{3+}$ & $\mathrm{Al}$ & $\mathrm{Mn}^{2+}$ & this work \\
Manganiandrosite-(Ce) & $\mathrm{Mn}{ }^{2+}$ & $\mathrm{Ce}$ & $\mathrm{Mn}^{3+}$ & $\mathrm{Al}$ & $\mathrm{Mn}^{2+}$ & Cenki-Toc et al. [10] \\
Manganiandrosite-(La) & $\mathrm{Mn}^{2+}$ & $\mathrm{La}$ & $\mathrm{Mn}^{3+}$ & $\mathrm{Al}$ & $\mathrm{Mn}^{2+}$ & Bonazzi et al. [19] \\
Uedaite-(Ce) & $\mathrm{Mn}^{2+}$ & $\mathrm{Ce}$ & $\mathrm{Al}$ & $\mathrm{Al}$ & $\mathrm{Fe}^{2+}$ & Miyawaki et al. [38] \\
Vanadoallanite-(La) & $\mathrm{Ca}$ & $\mathrm{La}$ & $\mathrm{V}^{3+}$ & $\mathrm{Al}$ & $\mathrm{Fe}^{2+}$ & Nagashima et al. [24] \\
Vanadoandrosite-(Ce) & $\mathrm{Mn}^{2+}$ & $\mathrm{Ce}$ & $\mathrm{V}^{3+}$ & $\mathrm{Al}$ & $\mathrm{Mn}^{2+}$ & Cenki-Toc et al. [10] \\
\hline
\end{tabular}

The first "akasakaite" to have been described was ferriakasakaite-(La) from a stratiform Fe-Mn ore deposit in the Shobu area, Ise City, Mie Prefecture (Japan) [2]. Actually, Cenki-Tok et al. [10] found an allanite group mineral having end-member formula $\mathrm{CaREEAlAlMn}{ }^{2+}\left(\mathrm{Si}_{2} \mathrm{O}_{7}\right)\left(\mathrm{SiO}_{4}\right) \mathrm{O}(\mathrm{OH})$, and Bonazzi et al. [39] studied a specimen from Kesebol (Sweden), corresponding to ferriakasakaite-(Ce), without proposing it as a new mineral species to the IMA-CNMNC. Following the first official description of an "akasakaite" by Nagashima et al. [2], Chukanov et al. [22] reported new occurrences of this mineral from the Laach Lake volcanic complex, Eifel (Germany) and the Pelagonian massif (Republic of North Macedonia).

The crystal chemical complexity shown by the allanite group minerals occurring in the Monte Maniglia Mn deposit likely reflects the variable geochemistry of the host rocks. For instance, the occurrence of phases having La or Ce has dominant REE may have different explanations, e.g., different $\mathrm{fO}_{2}$ conditions, in some cases reflecting the incipient oxidation of Ce to its tetravalent states and favoring the enrichment of La among REE, or it can reflect chemical inhomogeneity of the bulk rock. Finally, the strong zoning observed in the few studied grains of epidote supergroup minerals from Monte Maniglia may reflect a complex metamorphic-hydrothermal evolution of this small Mn deposit, suggesting the opportunity for more accurate studies to decipher its mineral assemblages and petrological evolution.

Supplementary Materials: The following are available online at http://www.mdpi.com/2075-163X/9/6/353/s1, CIF: manganiakasakaite-(La); ferriakasakaite-(Ce).

Author Contributions: C.B. (Corrado Balestra), R.B. and M.E.C. provided the studied specimens and performed preliminary analyses; C.B. (Cristian Biagioni) and F.Z. performed the experiments; C.B. (Cristian Biagioni), P.B., and M.P. analyzed the chemical and crystallographic data; C.B. (Cristian Biagioni), P.B., and M.P. wrote the paper with input from all the other authors.

Acknowledgments: We thank the University Centrum for Applied Geosciences (UCAG) for the access to the Eugen F. Stumpfl electron microprobe laboratory of Leoben (Austria). We also thank the four anonymous reviewers whose comments helped us to improve the paper.

Conflicts of Interest: The authors declare no conflict of interest. 


\section{References}

1. Armbruster, T.; Bonazzi, P.; Akasaka, M.; Bermanec, V.; Chopin, C.; Gieré, R.; Heuss-Assbichler, S.; Liebscher, A.; Menchetti, S.; Pan, Y.; et al. Recommended nomenclature of epidote-group minerals. Eur. J. Mineral. 2006, 18, 551-567. [CrossRef]

2. Nagashima, M.; Nishio-Hamane, D.; Tomita, N.; Minakawa, T.; Inaba, S. Ferriakasakaite-(La) and ferriandrosite-(La): New epidote-supergroup minerals from Ise, Mie Prefecture, Japan. Mineral. Mag. 2015, 79, 735-753. [CrossRef]

3. Bayliss, P.; Levinson, A.A. A system of nomenclature for rare-earth mineral species: Revision and extension. Am. Mineral. 1988, 73, 422-423.

4. Le Guernic, J. La zone du Roure: Contribution à l'étude du Briançonnais interne et du Piémontais en Haute Ubaye. Trav. lab. géol. Grenoble 1967, 43, 95-127.

5. Piccoli, G.C. Minerali delle Alpi Marittime e Cozie, Provincia di Cuneo; Amici del Museo "F. Eusebio": Alba, Italy, 2002; p. 370.

6. Piccoli, G.C.; Maletto, G.; Bosio, P.; Lombardo, B. Minerali del Piemonte e della Valle d'Aosta; Associazione Amici del Museo “F. Eusebio": Alba, Italy, 2007; p. 607.

7. Vanini, F.; Vignola, P.; Demartin, F.; Campostrini, I.; Rotiroti, N. Alleghanyite del Monte Maniglia, Val Varaita, Cuneo (Piemonte). MICRO 2019, 17, 111-112.

8. Mandarino, J.A. The Gladstone-Dale relationship. Part III. Some general applications. Can. Mineral. 1979, 17, 71-76.

9. Mandarino, J.A. The Gladstone-Dale relationship. Part IV. The compatibility concept and its application. Can. Mineral. 1981, 19, 441-450.

10. Cenki-Tok, B.; Ragu, A.; Armbruster, T.; Chopin, C.; Medenbach, O. New Mn- and rare-earth-rich epidote-group minerals in metacherts: manganiandrosite-(Ce) and vanadoandrosite-(Ce). Eur. J. Mineral. 2006, 18, 569-582. [CrossRef]

11. Kraus, W.; Nolze, G. PowderCell-A program for the representation and manipulation of crystal structures and calculation of the resulting X-ray powder patterns. J. Appl. Crystallogr. 1996, 29, 301-303. [CrossRef]

12. Bruker AXS Inc. Apex2; Bruker Advanced X-ray Solutions: Madison, WI, USA, 2004.

13. Bruker AXS Inc. Apex3; Bruker Advanced X-ray Solutions: Madison, WI, USA, 2016.

14. Sheldrick, G.M. Crystal structure refinement with SHELXL. Acta Crystallogr. 2015, C71, 3-8.

15. Wilson, A.J.C. (Ed.) International Tables for Crystallography. Volume C: Mathematical, Physical and Chemical Tables; Kluwer Academic: Dordrecth, The Netherlands, 1992.

16. Brese, N.E.; O'Keeffe, M. Bond-valence parameters for solids. Acta Crystallogr. 1991, B47, 192-197. [CrossRef]

17. Ferraris, G.; Ivaldi, G. Bond valence vs. bond length in O...O hydrogen bonds. Acta Crystallogr. 1988, B44, 341-344. [CrossRef]

18. Dollase, W.A. Refinement and comparison of the structure of zoisite and clinozoisite. Am. Mineral. 1968, 53, 1882-1888.

19. Bonazzi, P.; Menchetti, S.; Reinecke, T. Solid solutions between piemontite and androsite-(La), a new mineral of the epidote group from Andros Island, Greece. Am. Mineral. 1996, 81, 735-742. [CrossRef]

20. Dollase, W.A. Crystal structure and cation ordering of piemontite. Am. Mineral. 1969, 54, 710-717.

21. Bonazzi, P.; Garbarino, C.; Menchetti, S. Crystal chemistry of piemontites: REE-bearing piemontite from Monte Brugiana, Alpi Apuane, Italy. Eur. J. Mineral. 1992, 4, 23-33. [CrossRef]

22. Chukanov, N.V.; Zubkova, N.V.; Schäfer, C.; Varlamov, D.A.; Ermolaeva, V.N.; Polekhovsky, Y.S.; Jančev, S.; Pekov, I.; Pushcharovsky, D.Y. New data on ferriakasakaite-(La) and related minerals extending the compositional field of the epidote supergroup. Eur. J. Mineral. 2018, 30, 323-332. [CrossRef]

23. Bermanec, V.; Armbruster, T.; Oberhansli, R.; Zebec, V. Crystal chemistry of Pb- and REE-rich piemontite from Nezilovo, Macedonia. Schweiz. Mineral. Petrogr. Mitt. 1994, 74, 321-328.

24. Nagashima, M.; Nishio-Himane, D.; Tomita, N.; Minakawa, T.; Inaba, S. Vanadoallanite-(La): A new epidote-supergroup mineral from Ise, Mie Prefecture, Japan. Mineral. Mag. 2013, 77, 2739-2752. [CrossRef]

25. Nagashima, M.; Armbruster, T.; Akasaka, M.; Minakawa, T. Crystal-chemistry of $\mathrm{Mn}^{2+}{ }_{-}, \mathrm{Sr}_{-}$-rich and REE-bearing piemontite from the Kamisugai mine in the Sambagawa metamorphic belt, Shikoku, Japan. J. Mineral. Petrol. Sci. 2010, 105, 142-150. [CrossRef] 
26. Bonazzi, P.; Menchetti, S. Manganese in monoclinic members of the epidote group: Piemontite and related minerals. Rev. Mineral. Geochem. 2004, 56, 495-552. [CrossRef]

27. Dollase, W.A. Mössbauer spectra and iron distribution in the epidote group minerals. Z. Kristallogr. 1973, 138, 41-63. [CrossRef]

28. Ferraris, G.; Ivaldi, G. Manganese/iron distribution in a strontian piemontite by neutron diffraction. Z. Kristallogr. 1989, 187, 145-151. [CrossRef]

29. Langer, K.; Tillmanns, M.; Kersten, M.; Almen, H.; Arni, R.K. The crystal chemistry of $\mathrm{Mn}^{3+}$ in the clinoand orthozoisite structure type, $\mathrm{Ca}_{2} \mathrm{M}^{3+}{ }_{3}\left[\mathrm{OH}|\mathrm{O}| \mathrm{SiO}_{4} \mid \mathrm{Si}_{2} \mathrm{O}_{7}\right]$ : A structural and spectroscopic study of some natural piemontites and "thulites" and their synthetic equivalents. Z. Kristallogr. 2002, 217, 563-580.

30. Thomson, T. Experiments on allanite, a new mineral from Greenland. J. Nat. Phylos. Chem. Arts 1811, 29, 47-59. [CrossRef]

31. Orlandi, P.; Pasero, M. Allanite-(La) from Buca della Vena mine, Apuan Alps, Italy, an epidote group mineral. Can. Mineral. 2006, 44, 63-68. [CrossRef]

32. Škoda, R.; Cempírek, J.; Filip, J.; Novák, M.; Veselovský, F.; Čtvrtlík, R. Allanite-(Nd), $\mathrm{CaNdAl}_{2} \mathrm{Fe}^{2+}\left(\mathrm{SiO}_{4}\right)\left(\mathrm{Si}_{2} \mathrm{O}_{7}\right) \mathrm{O}(\mathrm{OH})$, a new mineral from Åskagen, Sweden. Am. Mineral. 2012, 97, 983-988. [CrossRef]

33. Nel, H.J.; Strauss, C.A.; Wickman, F.E. Lombaardite, a new mineral from the Zaaiplaats Tin Mine, Central Transvaal. Union South Afr. Dep. Mines Geol. Surv. Mem. 1949, 43, 45-57.

34. Grew, E.S.; Essene, E.J.; Peacor, D.R.; Su, S.-C.; Asami, M. Dissakisite-(Ce), a new member of the epidote group and the Mg analogue of allanite-(Ce) from Antarctica. Am. Mineral. 1991, 76, 1990-1997.

35. Tumiati, S.; Godard, G.; Martin, S.; Nimis, P.; Mair, V.; Boyer, B. Dissakisite-(La) from the Ulten zone peridotite (Italian Eastern Alps): A new end-member of the epidote group. Am. Mineral. 2005, 90, 1177-1185. [CrossRef]

36. Kartashov, P.M.; Ferraris, G.; Ivaldi, G.; Sokolova, E.; McCammon, C.A. Ferriallanite-(Ce), $\mathrm{CaCeFe}^{3+} \mathrm{AlFe}^{2+}\left(\mathrm{SiO}_{4}\right)\left(\mathrm{Si}_{2} \mathrm{O}_{7}\right) \mathrm{O}(\mathrm{OH})$, a new member of the epidote group: Description, $\mathrm{X}$-ray and Mössbauer study. Can. Mineral. 2002, 40, 1641-1648. [CrossRef]

37. Kolitsch, U.; Mills, S.K.; Miyawaki, R.; Blass, G. Ferriallanite-(La), a new member of the epidote supergroup from the Eifel, Germany. Eur. J. Mineral. 2012, 24, 741-747. [CrossRef]

38. Miyawaki, R.; Yokoyama, K.; Matsubara, S.; Tsutsumi, Y.; Goto, A. Uedaite-(Ce), a new member of the epidote group with Mn at the A site, from Shodoshima, Kagawa Prefecture, Japan. Eur. J. Mineral. 2008, 20, 261-269. [CrossRef]

39. Bonazzi, P.; Holtstam, D.; Bindi, L.; Nysten, P.; Capitani, G. Multi-analytical approach to solve the puzzle of an allanite-subgroup mineral from Kesebol, Västra Götaland, Sweden. Am. Mineral. 2009, 94, 121-134. [CrossRef]

(C) 2019 by the authors. Licensee MDPI, Basel, Switzerland. This article is an open access article distributed under the terms and conditions of the Creative Commons Attribution (CC BY) license (http://creativecommons.org/licenses/by/4.0/). 